\title{
HARDWARE IN THE LOOP TESTING OF MICROSATELLITE COMPONENTS
}

\author{
by Sarah Hardacre, Bachelor of Engineering \\ Ryerson University, Toronto, 2002
}

\author{
A thesis \\ presented to Ryerson University \\ in partial fulfillment of the \\ requirements for the degree of \\ Master of Applied Science \\ in the Program of \\ Mechanical Engineering
}

Toronto, Ontario, Canada, 2005

(C)Sarah Hardacre 2005 
All rights reserved

INFORMATION TO USERS

The quality of this reproduction is dependent upon the quality of the copy submitted. Broken or indistinct print, colored or poor quality illustrations and photographs, print bleed-through, substandard margins, and improper alignment can adversely affect reproduction.

In the unlikely event that the author did not send a complete manuscript and there are missing pages, these will be noted. Also, if unauthorized copyright material had to be removed, a note will indicate the deletion.

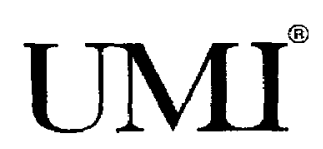

UMI Microform EC53021

Copyright 2008 by ProQuest LLC

All rights reserved. This microform edition is protected against unauthorized copying under Title 17, United States Code.

ProQuest LLC

789 East Eisenhower Parkway

P.O. Box 1346

Ann Arbor, Ml 48106-1346 


\section{Authors Declaration}

I hereby declare that I am the sole author of this thesis.

I authorize Ryerson University to lend this thesis to other institutions or individuals for the purpose of scholarly research.

I further authorize Ryerson University to reproduce this thesis by photocopying or by other means, in total or in part, at the request of other institutions or individuals for the purpose of scholarly research. 


\section{Borrower's Page}

Ryerson University requires the signatures of all persons using or photocopying this thesis. Please sign below, and give address and date.

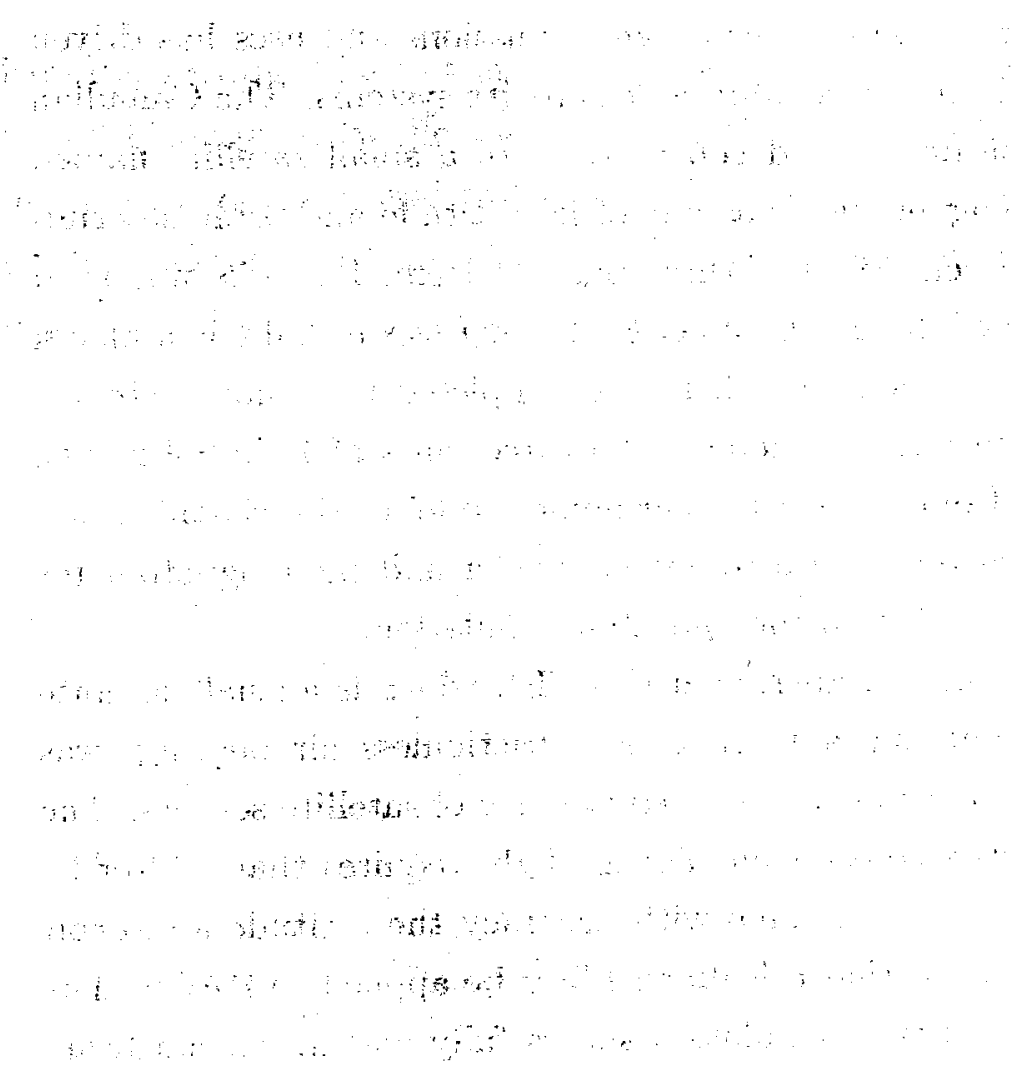




\begin{abstract}
.
Hardware in the Loop Testing of Microsatellite Attitude Control Components Master's of Applied Science, 2005
\end{abstract}

\title{
Sarah Hardacre
}

Mechanical Engineering

Ryerson University

The desire to bring space travel to a wider range of missions and uses has driven the market to using smaller and thus more affordable satellite systems. The Canadian Space Agency is completing the design and construction of a small satellite named QuickSat, which will utilize a magnetometer as one of its attitude and orbit determination instruments. A test bed comprised of three pairs of Helmoltz Coils was used for hardware in the loop testing of the magnetometer. Testing was initially completed to prove the capabilities of the test bed, and then was completed to demonstrate the capabilities of the flight qualified magnetometer. The three pairs of Helmholtz coils were driven by data calculated from a spherical harmonic model of the Earth's magnetic field. The coils were controlled using a robust controller and the magnetometer was used to drive the B-dot control law in the QuickSat simulation.

The Ryerson Attitude and Control Experiment (RACE), which is a small satellite sized platform, free to spin about one axis on a near frictionless air bearing, was utilized to develop and test a system to deal with redundancy of satellite sensors. The possibility of missing, noisy or erroneous output during flight requires that a filter be applied to a satellite's flight code to determine with accuracy the attitude and orbit of the spacecraft. It was thus decided that a Kalman Filter be applied to RACE. The Kalman filter was applied to the RACE simulation successfully and initial hardware testing was carried out. 


\section{Acknowledgements}

I would first like to thank my thesis advisor, DJ McTavish for his guidance and direction, as well as all his patience and support during the completion of this degree.

I would also like to show my appreciation to my advisors at the Canadian Space Agency, Alfred Ng and David Golla, for their help through out the completion of the QuickSat studies, as well as my co-workers, Christian Ribe and Suda Martins for their positive attitude during periods of discouragement and for all of the help they provided with technical details and programming issues.

Finally, I would like to thank my supervisor at the European Space Agency, Franco Ongaro, for all his support while I was writing this thesis. I have appreciated his constant encouragement and motivational talks, and the flexibility in scheduling that he allowed me to write while working full time. I am unable to express how much his understanding and constant positive spirit motivated me to keep working. 


\section{Contents}

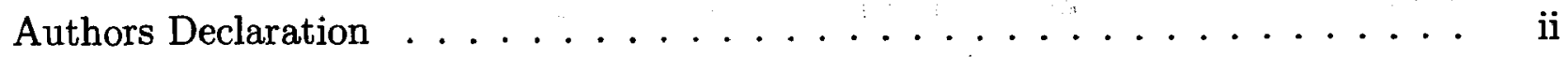

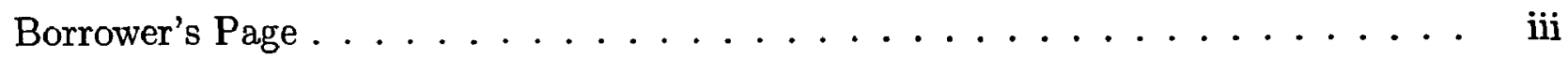

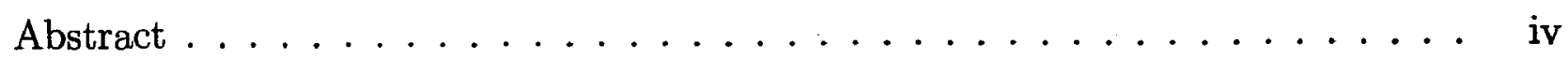

Acknowledgements ......................

Table of Contents ............................. vi

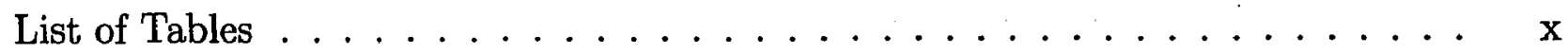

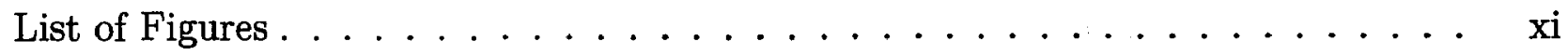

List of Symbols . . . . . . . . . . . . . . . . xiii

1 Introduction $\quad 1$

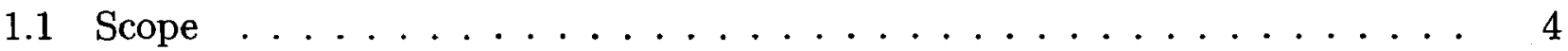

1.1 .1 QuickSat ........................ 4

1.1.2 Magnetometer Testing . . . . . . . . . . . . . . 4

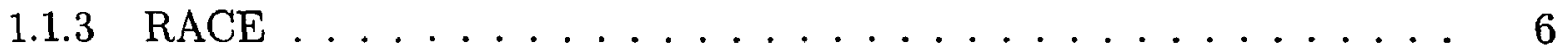

1.1.4 RACE Testing . . . . . . . . . . . . . . . 6

$\begin{array}{lll}2 & \text { Detailed System Descriptions } & 7\end{array}$

2.1 QuickSat. ........................... 7

2.1 .1 QuickSat Magnetometer . . . . . . . . . . . . 7

2.2 Description of the Helmholtz Cage $\ldots \ldots \ldots \ldots$ 
2.3 Support Structure . . . . . . . . . . . . . . . . . 10

2.4 Hardware and Software Components . . . . . . . . . . . . . 10

2.4 .1 Simulation .......................... 10

2.4 .2 Windows NT station $\ldots \ldots \ldots \ldots \ldots \ldots$

2.4 .3 QNX Station . . . . . . . . . . . . . 12

2.4.4 Data Acquisition and Control of Coils . . . . . . . . . . 12

2.5 RACE Components . . . . . . . . . . . . . . . . 13

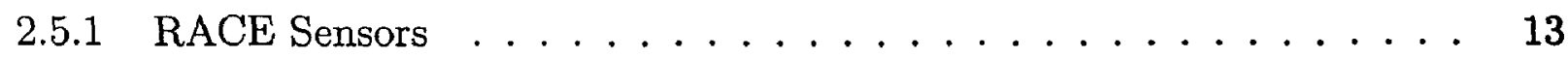

2.5 .2 Actuators .......................... 14

3 Modeling 15

3.1 QuickSat Modeling . . . . . . . . . . . . . . . 15

3.1 .1 System Dynamics . . . . . . . . . . . . . . 15

3.1 .2 Spacecraft Kinematics . . . . . . . . . . . . . 16

3.1 .3 Actuators . . . . . . . . . . . . . . 17

3.1 .4 Disturbance Torques . . . . . . . . . . . . . 18

3.1 .5 Orbital Mechanics . . . . . . . . . . . . . . 19

3.1 .6 Magnetic Field ... . . . . . . . . . . . . 20

3.2 RACE Modeling . . . . . . . . . . . . . . . 23

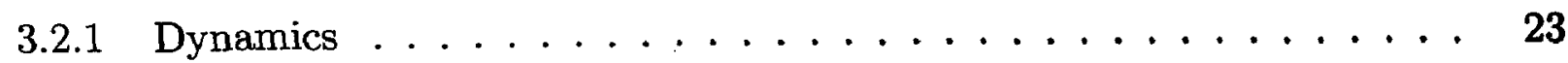

3.2 .2 State Space Model . . . . . . . . . . . . . 23 
3.2 .3 Momentum Wheel $\ldots \ldots \ldots \ldots \ldots$. . . . . . . . . 27

3.2 .4 Rate Sensor . . . . . . . . . . . . . . . 29

4 Controller Design for QuickSat Project $\quad 30$

4.1 Robust Controller . . . . . . . . . . . . . . . . . 30

4.2 B-dot Control Law . . . . . . . . . . . . . . . . . 34

5 Testing of Helmholtz Cage $\quad 38$

5.1 Verification of Magnetometer Measurements . . . . . . . . . . . 38

5.2 Axis Linearity and Coupling . . . . . . . . . . . . . . . . 39

5.3 Transfer Function . . . . . . . . . . . . . . . . . . . . 44

6 QuickSat Hardware in the Loop Simulation 46

6.1 Helmholtz Cage Controller Implementation . . . . . . . . . . . . . . . 46

6.1 .1 Misalignment of Magnetometers . . . . . . . . . . . . 47

6.2 Hardware in the Loop with QuickSat Simulation . . . . . . . . . . . . 49

6.2 .1 Open Loop Simulation . . . . . . . . . . . . . . . 49

6.2 .2 Closed Loop Simulation . . . . . . . . . . . . . . 52

6.3 B-dot Control Law . . . . . . . . . . . . . . . . 52

6.3.1 Stability of B-dot Control Law . . . . . . . . . . . . . . 54

7 Controller Design for RACE project $\quad 56$

7.1 PID Controller . . . . . . . . . . . . . . . . 56

7.2 Kalman Filter . . . . . . . . . . . . . . . 56 
8 RACE Simulations $\quad 61$

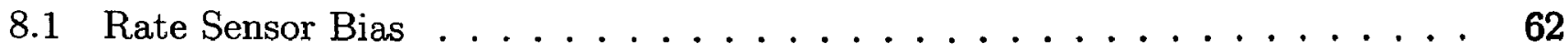

8.2 Controller Testing ....................... 65

8.3 Kalman Filter Testing ...................... 66

9 Concluding Remarks $r$

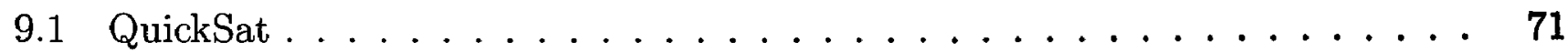

9.2 RACE ........................... 72

9.3 Recommendations for Future Work . . . . . . . . . . . . . . . 72

$\begin{array}{lr}\text { References } & \mathbf{7 3}\end{array}$ 


\section{List of Tables}

1 Helmholtz Coil Characteristics . . . . . . . . . . . . . . . . . . 10

2 Friction Coefficients . . . . . . . . . . . . . . . . 28

3 Static Magnetic Field Measurements At Center of Helmholtz Cage . . . . . 38

4 Billingsley Scaling Factor . . . . . . . . . . . . . . . . 39

5 Values of Coil Gains and Their Associated $R^{2}$ Values $\ldots \ldots \ldots \ldots$

6 Time Constants for the Coil System . . . . . . . . . . . . . . . 45

7 Robust Controller Gains for Helmholtz Cage . . . . . . . . . . . . . . 46

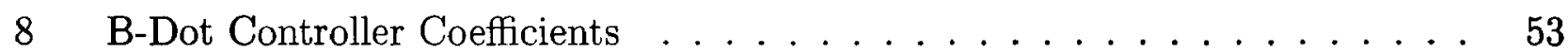

9 Various Initial Tumbling Rates and their Corresponding Time to Reach Mode

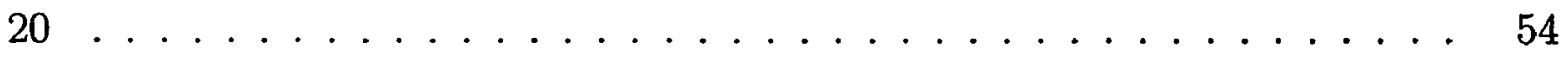

10 Robust Controller Gains for Helmholtz Cage . . . . . . . . . . . . . . . . 66 


\section{List of Figures}

1 QuickSat Model ........................ 5

2 Ryerson Satellite Attitude Control Experiment (RACE) $[16] \ldots \ldots \ldots$

3 Magnetometers Used for QuickSat (above:Billingsly, below:Barington) . . . 8

4 Helmholtz Coil Configuration . . . . . . . . . . . . . . . 9

5 The Support Structure Holding the Magnetometers in the Centre of the Coil Configuration .......................... 11

6 The Hardware in the loop System . . . . . . . . . . . . . . . . 13

7 Definition of Orbital Parameters . . . . . . . . . . . . . . . 20

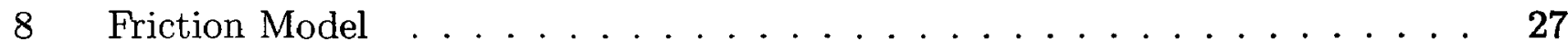

9 Angluar Velocity of RACE Momentum Wheel in the presence of friction only with initial rate of rotation of $15 \% \ldots \ldots \ldots 28$

10 Robust Controller Block Diagram . . . . . . . . . . . . . . . . . . 35

11 Relationship between Input Current Along X-Axis and Output Voltage of Magnetometer . . . . . . . . . . . . . . . . . .

12 Relationship between Input Current Along Y-Axis and Output Voltage of Magnetometer . . . . . . . . . . . . . . . . . .

13 Relationship between Input Current Along Z-Axis and Output Voltage of Magnetometer . . . . . . . . . . . . . . . . . . 42

14 Response Along the X-Axis to a Step Input $\ldots \ldots \ldots$. . . . . . . . 43

15 Response Along the Y-Axis to a Step Input . . . . . . . . . . . . . . 43

16 Response Along the Z-Axis to a Step Input . . . . . . . . . . . . . . . 44 
17 Circuit for determining the Time Constant of the coils . . . . . . . . 45

18 Response of the Coils to Sine Wave Input, Measured with the Barington Magnetometer . . . . . . . . . . . . . . . . . . .

19 Response of the Coils to sine Wave Input, Measured with the Billingsly Magnetometer . . . . . . . . . . . . . . . . 48

20 Error in Magnitude of Field with sine Input Alone Each Axis . . . . . . . 50

21 Magnitude of Field . . . . . . . . . . . . . . . . . 51

22 Magnitude of Field . . . . . . . . . . . . . . . . . . 52

23 Response of Each Axis to Simulation Input With Billingsly Magnetometer Data Driving Simulation . . . . . . . . . . . . . . . 53

24 Magnitude of the Magnetic Field with Simulation Running until Mode 70 . . 55

25 Summary of Kalman Filter . . . . . . . . . . . . . . . . . . . . 60

26 Rate Sensor Readings while RACE is fixed and actuators are powered off . . 62

27 Rate Sensor Readings while RACE is fixed and actuators are powered. . . . 63

28 Effects of Increased Temperature on Rate Sensor . . . . . . . . . . . . . . 64

29 Angular Rates of a free spinning RACE platform . . . . . . . . . . . 65

30 Response of RACE to a Step Input . . . . . . . . . . . . . . . . . 67

31 Kalman Filter Estimates of Angular Position (Sampling Frequency of the encoder $=0.2 \mathrm{~Hz}$ ), Angular Position Not Normalized to $2 \pi \ldots \ldots 69$

32 Kalman Filter Estimates of Angular Velocity (Sampling Frequency of the

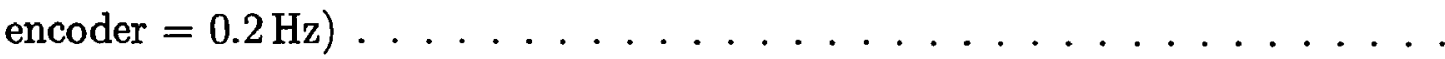




\section{List of Symbols}

\begin{tabular}{ll}
$a$ & Inverse of Time Constant \\
$a$ & Semi-Major Axis \\
$a$ & Equatorial Radius of Earth \\
$\mathbf{A}$ & Error Space State Matrix \\
$A_{p s}$ & Area Projected Normal to the Sun \\
$\mathbf{B}$ & Magnetic Field \\
$\mathbf{B}$ & Error Space Feedback Matrix \\
$\mathbf{B}_{d e s}$ & Desired Magnetic Field Along Each Axis of Coils \\
$\mathbf{C}$ & Orientation Matrix \\
$C_{d}$ & Drag Coefficient \\
$e$ & Eccentricity \\
$\mathbf{e}$ & State Error Vector \\
$\mathbf{e}_{k}^{+}$ & State Error Vector Directly After State Measurement (Kalman Filter) \\
$\mathbf{e}_{k}^{-}$ & State Error Vector Directly Before State Measurement (Kalman Filter) \\
$E$ & Kinetic Energy \\
$\mathbf{F}$ & System Matrix \\
$\mathbf{F}_{a}$ & Aerodynamic Force \\
$\mathbf{F}_{a}$ & Solar Radiation Force \\
$g_{n}^{m}, h_{n}^{m}$ & Gaussian Coefficients \\
$g^{n m}, h^{n m}$ & Schmidt Normalized Gaussian Coefficients \\
$\mathbf{g}$ & Net Torque Acting on Wheel Plus Platform \\
$g_{o}, \omega_{o}, b$ & Friction Model Parameters \\
$\mathbf{g}_{w}$ & Net Torque Acting on Wheel \\
$\mathbf{G}$ & Input Matrix \\
$G(s)$ & Transfer Function \\
$\mathbf{h}$ & Angular Momentum \\
$\mathbf{H}$ & Measurement Matrix \\
$i_{x y z}$ & Current in Coils \\
$i$ & Orbital Inclination \\
& \\
\hline &
\end{tabular}




\begin{tabular}{|c|c|}
\hline$I$ & Inertia \\
\hline$I_{B}$ & Inertia of Spacecraft Without Including Wheel \\
\hline$I_{W}$ & Inertia of Reaction Wheel \\
\hline$K$ & Spacecraft Reflectivity \\
\hline $\mathrm{K}_{k}$ & Kalman Filter Gain \\
\hline $\mathbf{K}$ & Robust Controller Gain \\
\hline$K_{d}$ & PID Derivative Gain \\
\hline$K_{i}$ & PID Integral Gain \\
\hline$K_{p}$ & PID Proportional Gain \\
\hline$L$ & Inductance \\
\hline $\mathbf{m}$ & Dipole Moment \\
\hline$\hat{\mathbf{n}}$ & Satellite Axis of Rotation \\
\hline$\tilde{\mathbf{q}}=$ & Euler Parameters \\
\hline$\hat{\mathbf{n}}$ & Nadir Vector \\
\hline $\mathbf{P}_{k}^{+}$ & Covariance Matrix Directly After Measurement (Kalman Filter) \\
\hline $\mathbf{P}_{k}^{-}$ & Covariance Matrix Directly Before Measurement (Kalman Filter) \\
\hline $\mathbf{P}_{k+1}^{-}$ & Covariance Matrix Directly After State Propagation Step (Kalman Filter) \\
\hline$P_{n}^{m}$ & Legendre Polynomials \\
\hline$P^{n m}$ & Schmidt Normalized Legendre Polynomials \\
\hline$P_{s}$ & Solar Pressure \\
\hline $\mathbf{Q}_{k}$ & Covariance of process noise \\
\hline$r$ & Distance from Center of Earth \\
\hline $\mathbf{r}$ & Reference Signal \\
\hline $\mathbf{r}$ & Vector from the Center of Mass to the Optical Center of Pressure \\
\hline $\mathbf{r}_{c p}$ & Center of Pressure Vector \\
\hline$R$ & Resistance \\
\hline $\mathbf{R}_{k}$ & Covariance of Process Noise \\
\hline$S$ & Spacecraft Projected Area \\
\hline$t$ & Time \\
\hline$t_{p}$ & Time of Periapsis Passing \\
\hline
\end{tabular}




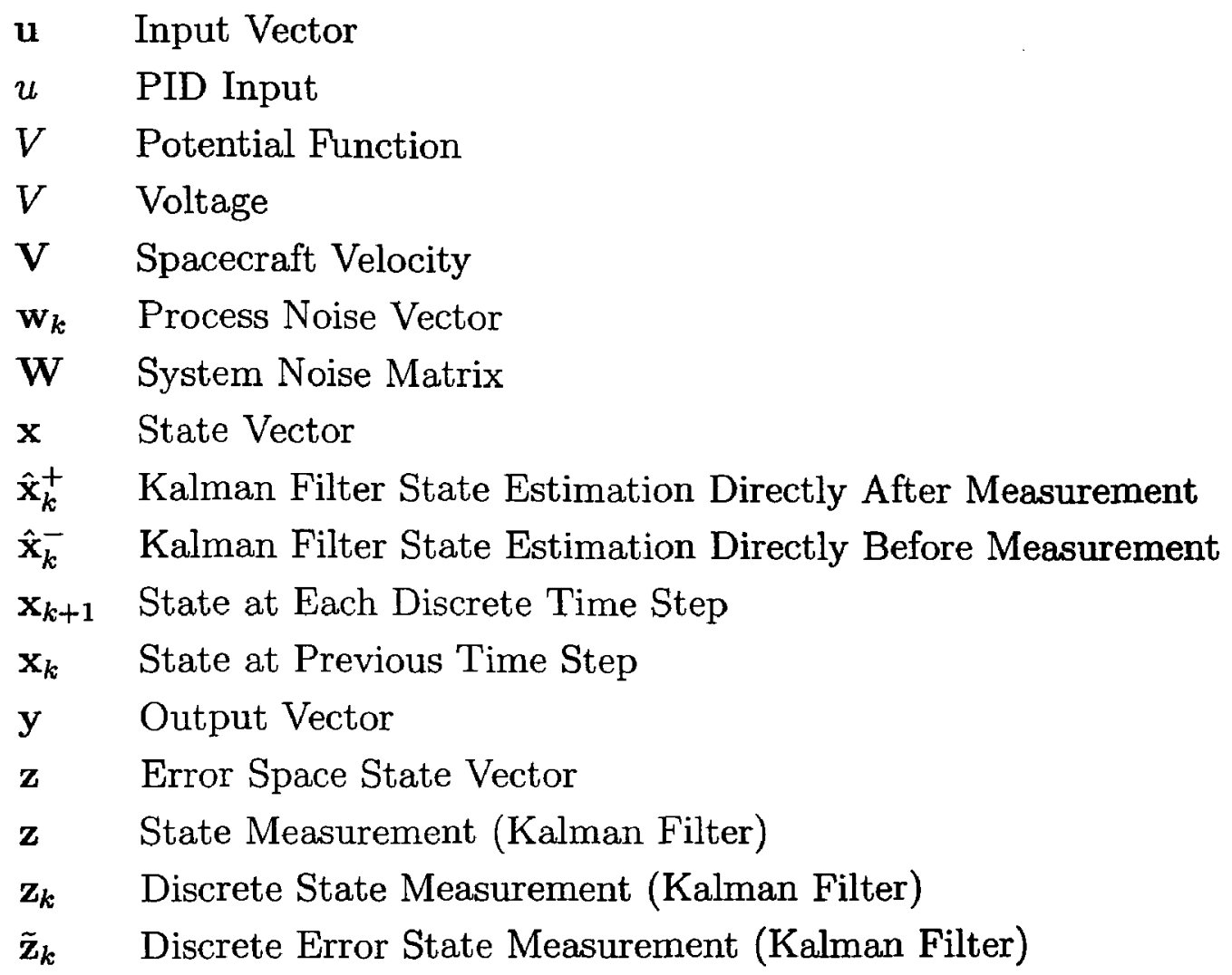




$\begin{array}{ll}\alpha_{1}, \alpha_{2} & \text { Reference Signal Parameters } \\ \beta & \text { Rate Sensor Bias } \\ \delta & \text { Kronecker Delta Function } \\ \eta & \text { Noise Term } \\ \theta & \text { Coelevation } \\ \theta & \text { Angular Position } \\ \boldsymbol{\mu} & \text { Error Space Input Vector } \\ \nu_{\boldsymbol{k}} & \text { Measurement Noise Vector } \\ \boldsymbol{\xi} & \text { Error Space State Vector } \\ \rho & \text { Atmospheric Density } \\ \tau_{a} & \text { Aerodynamic Torque } \\ \tau_{d i s t} & \text { Disturbance Torque } \\ \tau_{e x t} & \text { External Torque } \\ \tau_{G} & \text { Gravity Gradient Torque } \\ \tau_{m a g} & \text { Magnetic Torque } \\ \boldsymbol{\tau}_{s} & \text { Solar Radiation Torque } \\ \phi & \text { State Transition Matrix } \\ \phi & \text { Satellite Angle of Rotation } \\ \phi & \text { East Longitude from Greenwich } \\ \omega & \text { Angular Velocity } \\ \omega & \text { Frequency of a sine wave } \\ \omega & \text { Argument of the Periapsis } \\ \Omega & \text { Argument of the Ascending Node } \\ \omega_{o} & \text { Orbital Rate } \\ \omega_{W} & \text { Angular Velocity of Reaction Wheel }\end{array}$




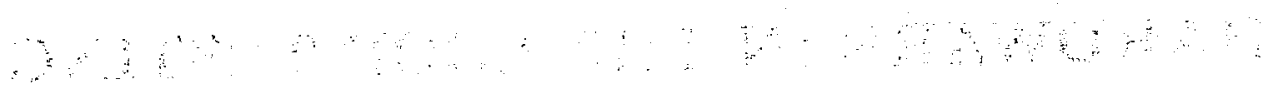

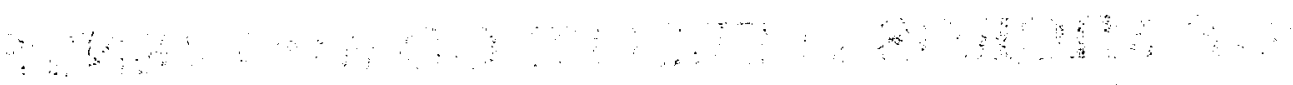

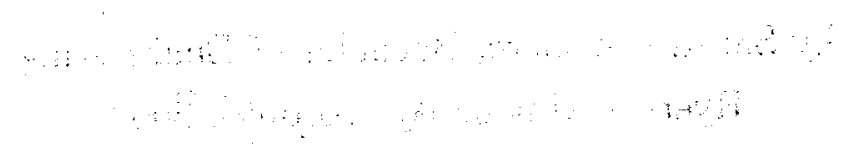

a

- $\quad \therefore \quad \cdots$

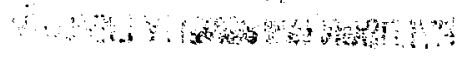




\section{Introduction}

The use of small and microsatellites for Earth observation, communications, navigation and science missions is increasing. It is generally accepted that small satellites have a mass less than $1000 \mathrm{~kg}$, anything below $250 \mathrm{~kg}$ is considered a microsatellite and anything less than 10 $\mathrm{kg}$, a nanosatellite. Advancing technology in domains such as electronics, computer systems, and material science have made small satellites a viable option for space based solutions. The smaller platforms offer advantages such as shorter design periods and lower mass to reach orbit, leading to, in many situations, significantly lower costs [5].

There are several programmes in flight and many planned that have utilized small satellite designs to complete their mission objectives. The European Galileo Programme, for example, will be implementing a global navigation system, similar to the American GPS, which will use a constellation of thirty small satellites (mass of approximately $670 \mathrm{~kg}$ ).

Orstead is a satellite with launch mass of $62 \mathrm{~kg}$ that was put into orbit February 23, 1999. This small satellite carried a payload that precisely mapped the magnetic field of the Earth, measured the charged particle environment and collected Global Positioning System (GPS) occultation data [26].

These relatively small satellites require unique miniaturized onboard systems, such as propulsion, electric and computer systems that have reduced mass, reduced volume and demand minimal power consumption when compared to their predecessors.

The attitude and orbit determination and control system (ACS) is of particular interest as it can be the limiting factor to the applicability of small satellites to certain missions. Small satellites tend to use passive attitude control devices to minimize mass and power consumption as well as increase simplicity of design which lowers cost. Rigid booms, for example, are used to achieve gravity gradient stabilization [10]. Passive attitude control often lacks the pointing accuracy required for many project objectives. The development of rehable miniaturized electronics and low cost computational capabilities has led to the movement towards using active stabilization onboard microsatellites. This allows for higher pointing accuracy which in turn increases the range of missions that can be fulfilled using 


\section{small satellites.}

Active control can be achieved by using magnetic torquers which interact with the Earth's magnetic field to create and control torque. These actuators have low mass, require low power consumption and are reliable due to their simplicity of design. Active magnetic control has been proposed for satellites ranging in mass from 40 to $200 \mathrm{~kg}$ in low Earth orbits with minimum inclinations of $28.5^{\circ}[10]$. Although active magnetic control is possible on its own, generally magnetic torquers are used in combination with reaction or momentum wheels to increase reliability and pointing accuracy.

The momentum wheel and magnetic torquer configuration was used onboard the Canadian satellite, MOST (Microvariability and Oscillation of Stars), which was launched in June, 2003 and has now completed over a year of its mission successfully. MOST was designed to monitor tiny light variations that are undetectable from Earth in stars and extra-solar planets. A similar actuator configuration was also used on Odin, a Swedish satellite which was launched February 20, 2001 for observations of the Earth's atmosphere and astronomy. As of early 2003, Odin had completed its two year design-goal lifetime successfully [24]. Another example is ChipSat, the Cosmic Hot Interstellar Plasma Spectrometer, which was launched January 13, 2003 and utilized four momentum wheels and three coils torquers to maintain its desired orientation.

Accurate satellite pointing can only be carried out if the orientation of the satellite and any error that may be present are known. A variety of sensors have been developed for use on satellites to enable the determination of satellite orientation. Satellites can be equipped with sun sensors, which determine the satellites position by measuring the angle at which the sunlight hits their photosensitive surfaces, Earth horizon sensors that use the thermal differences between the Earth (hot) and space (cold) to determine the orientation of the satellite, as well as magnetometers that measure the magnetic field and compare it to an onboard model to determine orientation and orbit.

The magnetometer is lightweight, compact, reliable and robust, making it an ideal choice for microsatellites. It has been shown that satellites equipped with magnetic control algorithms can converge to a stable state with initial errors of hundreds of kilometers and unknown 
attitude as quickly as a couple of orbits [13]. The magnetometer is dependent on an accurate model of the Earth's magnetic field and measurements can be affected by magnetic storms and surrounding electronic and metallic equipment. Even so, there are studies that have shown accuracies in positioning within $1-2 \mathrm{~km}$ and 0.25 degrees using only magnetometer data [13].

Many satellites have taken advantage of the Earth's magnetic field for attitude determination. Two Czech missions, APEX and Active, had components (or subsatellites) Magion-2 and Magion-3 respectively, that used magnetometers as the sole source of attitude information. These satellites showed an orientation determination accuracy of $5^{\circ}[6]$.

To increase and ensure accuracy, satellites are generally equipped with a variety of sensors. The increased amount of input data to the systems flight code can prove to be a challenge to process. Multiple sensors are often reading the same information with variable degrees of accuracy and the flight code must be able to compute an estimate that is based on all the information. Moreover, sensors can be susceptible to external perturbations, such as abnormal fluctuations in the electrical equipment or solar flares, and certain sensors cannot output information throughout the entire mission; for example, sun sensors cannot provide measurements during eclipse periods. Hence, the flight code must be able to handle redundant, possibly erroneous, missing and noisy data while still providing an accurate estimate of the spacecraft attitude and orbit.

One algorithm that is used regularly among satellite control system designers to estimate the system properties is the Kalman filter. The Kalman filter was first developed in 1960 by R.E. Kalman as a way to handle problems that involve the separation of random signals from random noise and the detection of signals of known form in the presence of random signals [15].

This filter uses a dynamical model for the time development of the system and a model of the sensor measurements to obtain the most accurate estimate possible of the system states using a linear estimation based on present and past measurements [15].

The Kalman filter was initially used in linear estimation, but was soon adapted for nonlinear orbital guidance and navigation problems, showing impressive results as early as 1967 [17]. 


\subsection{Scope}

The work described in this thesis involves testing of hardware and software components two different small satellite systems. First, a magnetometer for a microsatellite project, QuickSat, was tested, and then a Kalman filter was developed to be used in a small satellite test facility, RACE.

\subsubsection{QuickSat}

In 1998, the Canadian Space Agency began the development of a small satellite engineering model called QuickSat. The QuickSat project was a precursor to Canada's small and microsatellite program, which was established to provide a low cost means for Canadian industries to put their technology in space, as well as to build the skills necessary for small satellite development within the Canadian workforce [21]. To take advantage of the experience gained while developing MOST, and to benefit from well proven technologies, it was decided that QuickSat would be equipped with an attitude and control system that is comprised of a magnetometer, sun sensors, horizon sensors, a momentum wheel and magnetic torquers.

The primary payload of QuickSat is an amateur relay between earthbound HAM radio operators and the International Space Station. A secondary module was developed to support variable payloads of mass up to $25 \mathrm{~kg}$. This will allow QuickSat to be functional for a variety of tasks and missions.

The research and development of QuickSat has thus far improved internal expertise in many disciplines and domains, such as electronics, flight code, and attitude control.

\subsubsection{Magnetometer Testing}

As part of the thesis work, the magnetometer that will be flown aboard QuickSat was tested. This was done by using a test bed at the Canadian Space Agency, in conjunction with the QuickSat simulation for hardware in the loop testing. 


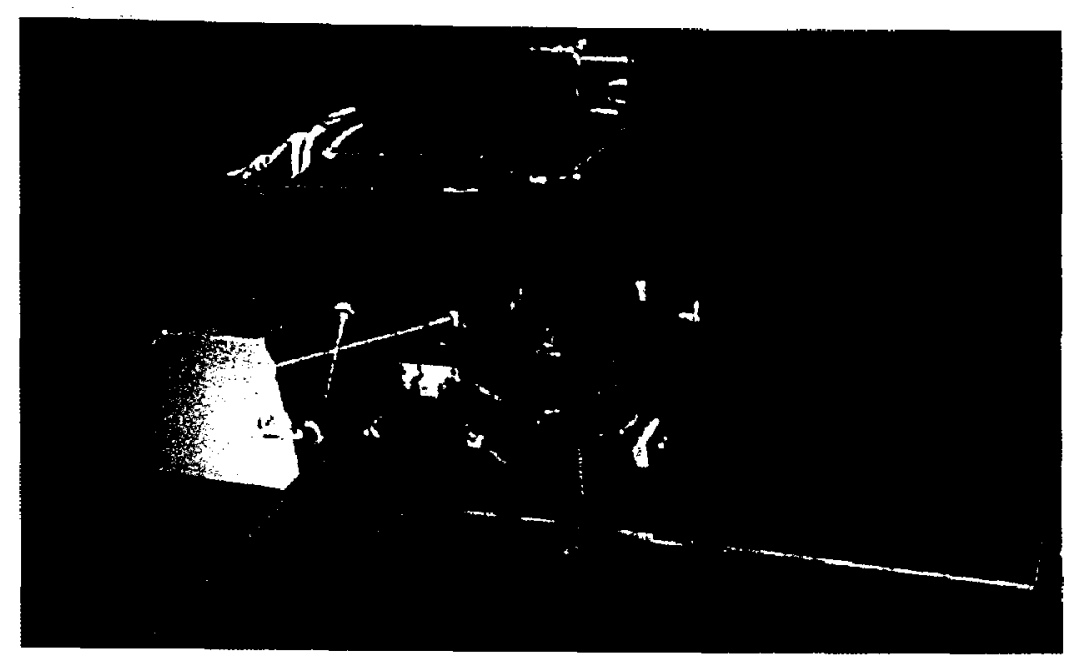

Figure 1: QuickSat Model

Characterization of the coils was carried out to determine the axis linearity and independence. The transfer function was determined experimentally for each axis by determining each time constant simultaneously, so that accurate modeling could be completed and proper control parameters could be chosen.

A robust controller was applied to the Helmholtz coils to ensure that the desired magnetic fields could be created. The controller was tested, and it was shown that the coils could produce a magnetic field equivalent to one a magnetometer would observe in orbit.

Once control of the test bed was achieved, the second magnetometer, the flight-rated instrument, was added to the set-up. Static measurements - the magnetic field was held constant - were taken to calibrate the magnetometer. Following determination of the proper magnetometer gain, the magnetometer was then integrated into the hardware in the loop simulation, first just taking measurements, and then fully integrated to drive the attitude control system within the QuickSat flight code.

Finally, the QuickSat flight code was altered to test a B-dot controller during hardware in the loop simulations. Several different free tumbling rates - considered as the initial conditions of the simulation - were tested. 


\subsubsection{RACE}

The Ryerson Satellite Attitude and Control Experiment, RACE, is a mock satellite system used by undergraduate and graduate students for research in dynamics and control. RACE sits on a near frictionless air bearing and is free to rotate in one dimension.

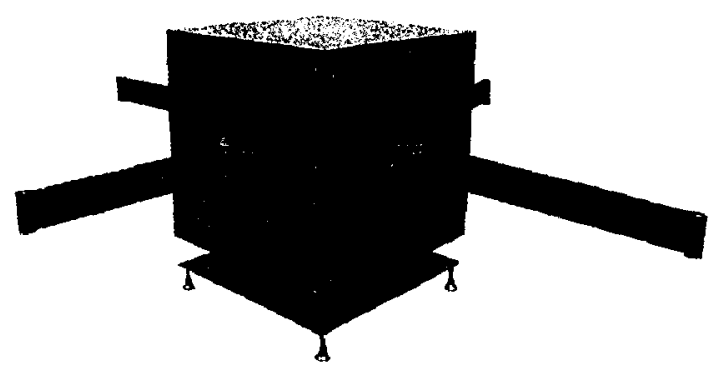

Figure 2: Ryerson Satellite Attitude Control Experiment (RACE)[16]

RACE is capable of running in two configurations, as a rigid structure, or as a rigid structure with the possibility of one to four flexible appendages - designed to simulate the solar panels present on most microsatellites. For the purposes of this study, RACE will only be considered in the rigid structure configuration. RACE is battery operated making the system capable of running as an independent unit. The computer-based control system collects data and controls angular position and rate.

To enhance the facility's capabilities, making it further resemble an actual microsatellite system, a Kalman filter was designed and implemented.

\subsubsection{RACE Testing}

A simulation of the RACE test equipment was completed and a Kalman filter was implemented showing the theoretical capability of the system to handle inconsistent and erroneous data. Initial hardware testing was carried out, including testing of the onboard sensors, testing of the controller and testing of the Kalman filter while RACE was spinning freely and while false initial conditions were applied. 


\section{Detailed System Descriptions}

\subsection{QuickSat}

QuickSat is a microsatellite, which contrary to most satellite designs was not constructed around its payload, but was intended to be a generic satellite capable of handling various payloads. It is designed to have a mass of less than $80 \mathrm{~kg}$, while supporting a payload of up to $30 \mathrm{~kg}$.

\subsubsection{QuickSat Magnetometer}

QuickSat is three-axis stabilized using sun sensors (six coarse resolution and two medium resolution), earth horizon sensors (two), a magnetometer, a momentum wheel and three magnetorquers.

The magnetometer, the instrument of interest in this study, to be used onboard Quicksat is a Billingsley TFM100G2, which is can measure magnetic fields in the range of -1 Gauss to +1 Gauss. It is a miniature tri-axial magnetometer, which consists of three magnetic sensors operating independently and simultaneously. Each of the three sensors has an analog output relative to the magnetic field present along its axis.

The magnetometer has a mass of $100 \mathrm{~g}$ and dimensions of $3.51 \mathrm{~cm} \times 3.23 \mathrm{~cm} \times 8.26 \mathrm{~cm}$. It has low mass and is compact, ideal for use onboard a microsatellite. Refer to Figure 3.

One of the objectives of this study was to test this magnetometer to determine its capabilities onboard QuickSat.

To eliminate the need for overly complicated robotics, or moving parts of any kind, a combination of Helmholtz coils was used. A Helmholtz coil is an electric wire wrapped around two identical rings separated co-axially by a constant distance equivalent to its radius. This configuration can produce a homogenous magnetic field at its geometric center, where the magnetometer will be placed for testing. This type of apparatus has been widely used for testing magnetometers, and these cages are commercially available. 


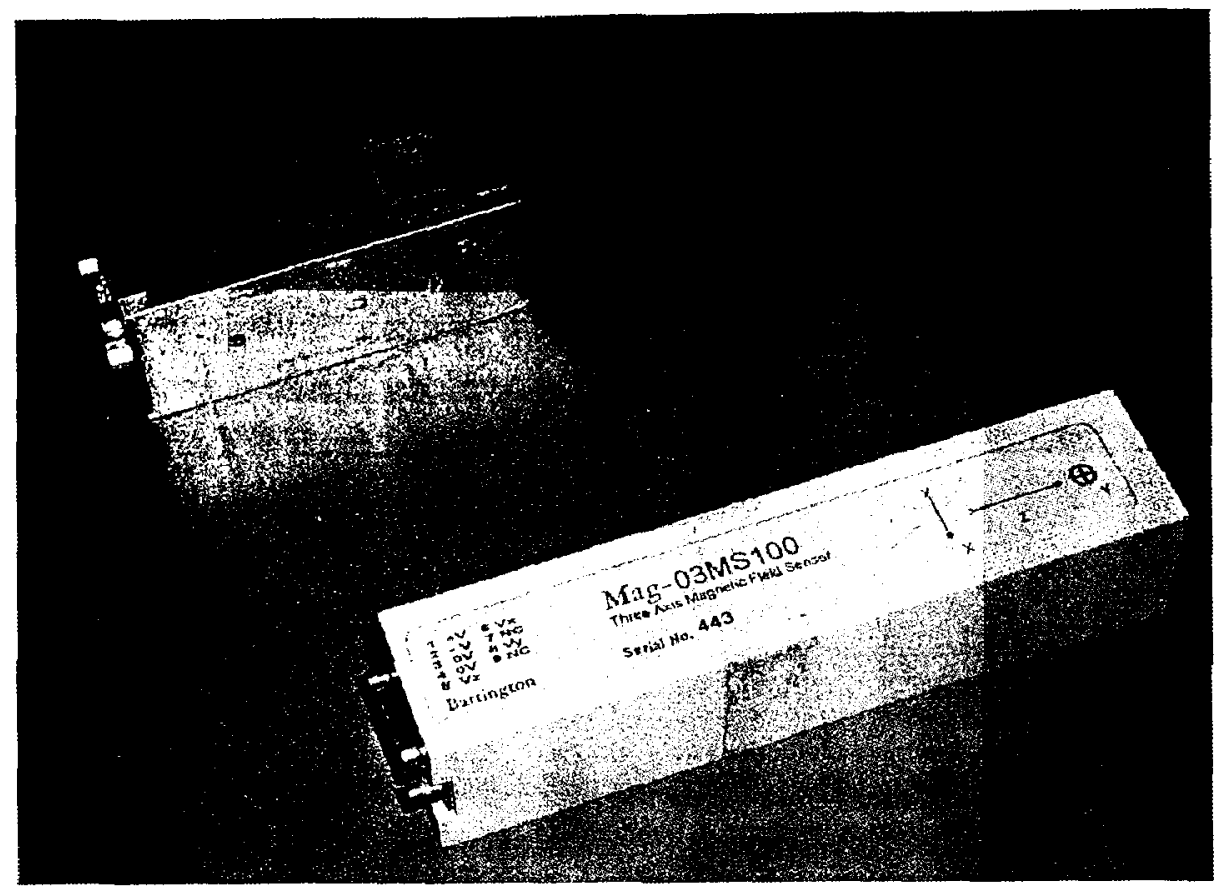

Figure 3: Magnetometers Used for QuickSat (above:Billingsly, below:Barington)

The Helmholtz Cage output was monitored by a separate non-space rated magnetometer, a Barington MAG-03MS. This magnetometer has a detectable range of $\pm 1 \mathrm{G}$ and an orthogonal error of $\pm 0.1^{\circ}$ (as specified by the manufacturer).

\subsection{Description of the Helmholtz Cage}

The coils were designed and constructed at the Canadian Space Agency in 1999 by Steve Marchetti [18] to create a magnetic field of $\pm 1 \mathrm{G}$ while taking into account the Earth's magnetic field at the location of the test bed. This value was chosen because it is equivalent to the measurement range of the magnetometer.

Three pairs of Helmholtz coils were erected at $90^{\circ}$ to each other to be able to control the magnetic field on three axes. Traditional Helmholtz Coils consists of two identical circular rings of coils; however, it was decided to simplify the construction of the system by building 


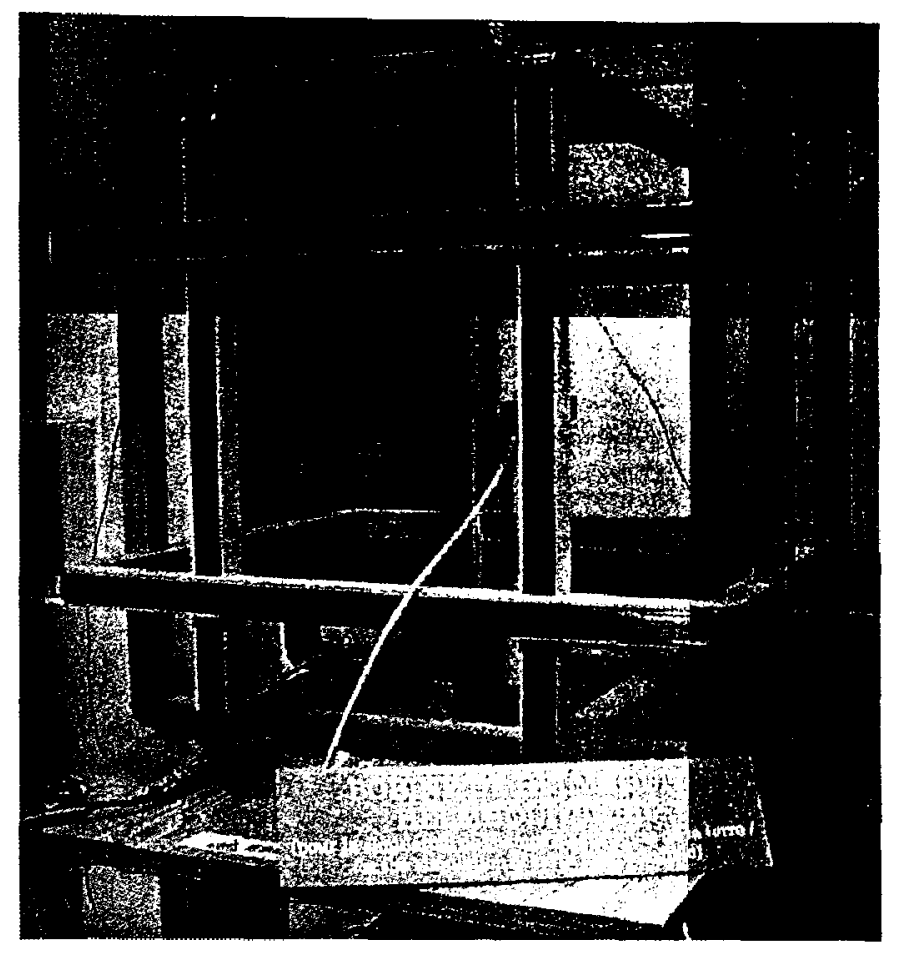

Figure 4: Helmholtz Coil Configuration

identical square rings. This simplification in construction did not compromise the homogeneity of the magnetic field at the geometric center of the coils, the area of interest for testing.

All the items used to construct the coils were made of non-magnetic material to eliminate interference caused by the structure during testing. The frame was assembled using dried hardwood, with wooden dowels at the corners and glue to hold everything together.

The wire was chosen to produce the maximum magnetic field with $1 \mathrm{~A}$ of current,, thereby limiting excess heat creation and allowing the use of a common off the shelf power supply. The coils were wrapped in two layers, which was found to provide enough strength in magnetic field without reducing the size of the homogenous field at the center of the coils. Properties of the coils can be found in Table 1.

The design and construction of the Helmholtz cage was completed in 1999, and initial testing 


\begin{tabular}{|c|c|c|c|}
\hline Coil Pair & Length of Wire $[\mathrm{cm}]$ & Wire Weight $[\mathrm{kg}]$ & Resistance $[\Omega]$ \\
\hline 1 & 362 & 1.35 & 7.73 \\
2 & 376 & 1.40 & 8.03 \\
3 & 390 & 1.46 & 8.33 \\
\hline
\end{tabular}

Table 1: Helmholtz Coil Characteristics

on the system began in 2000 .

\subsection{Support Structure}

A secure system for suspending both the magnetometers in the geometric center of the coils was designed and constructed as one was not previously available. This device was made of wood and plastic so that the surrounding electric current and magnetic fields would not be altered. The structure ensures that if dismantling of the system were necessary, the two magnetometers could be returned to the same position relative to each other and the coils during reassembly so that minimal calibration would be necessary to restart testing. Refer to Figure 5.

\subsection{Hardware and Software Components}

Many hardware and software components were necessary for hardware in the loop testing, to control the Helmholtz Cage and access information from the magnetometers. These include a simulation created in Simulink, two computer workstations, both running versions of the same simulation, and data transfer hardware.

\subsubsection{Simulation}

During the QuickSat design phase, a simulation analysing the orbiting satellite was developed in MatLab Simulink by a team of engineers and software experts. This simulation was already 


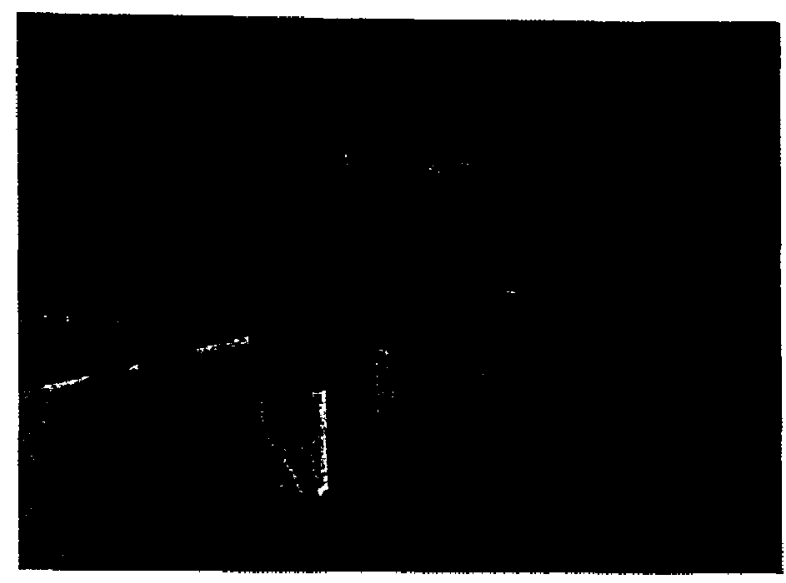

Figure 5: The Support Structure Holding the Magnetometers in the Centre of the Coil Configuration

in place and undergoing validation when coil testing began, and was utilized for the hardware in the loop experiments. The magnetic field that the satellite would experience was one of the many calculated values in the simulation, and this data was used as the desired magnetic field that needed to be created by the coils.

The simulation was capable of communicating with hardware components in real time. A software package, RT Lab was used for real time simulations in conjunction with the Simulink package. The simulation was able to send information to the power sources driving the Helmholtz coils, as well as receive information from the magnetometers.

\subsubsection{Windows NT station}

The Windows NT workstation was the user interface for the Helmholtz coils. Experimental data was analyzed and logic was initiated on this workstation. The models and control algorithms were built in MatLab Simulink, and the simulation was sent to the Opal-RT software to be compiled and run in real time. 


\subsubsection{QNX Station}

A second station was used that had a QNX real-time operating system. This type of software allows the simulation to react to inputs in real time and thus simulate the events as they would actually occur. Control over the simulation (start, stop etc.) lies on the Windows platform; however, the real-time simulation itself was running independently of user interface simulation. Data was passed back to the Windows station during simulation so that the user could assess the information qualitatively through graphs, counters and numerical displays during the tests. However, the real time data was stored on the QNX station and was retrieved for analysis after the test was completed. The power supplies powering each axis of the Helmholtz cage received their instructions from the real time simulation running on the QNX system.

\subsubsection{Data Acquisition and Control of Coils}

Each coil pair was powered with an HP6612C System DC power supply with a maximum output of $2 \mathrm{~A}$. The polarity of the power supplies was controlled with an external mechanical relay circuit.

A general purpose interface bus (GPIB) was used to supply the coil power sources with commands. An AT-GPIB/TNT fits into the ISA slot of any standard personal computer and has a $20 \mathrm{~ms}$ delay which was deemed to have negligible effect on the results of the tests. It also has a digital output line, which is used to trigger the mechanical relay that controls the polarity of the coil power sources.

A PCI-MIO-16XE-10 data acquisition card (DAQ) was used to read the magnetometer signals, and send these values back to the QNX system for simulation purposes and then to the user interface for qualitative examining of the data during the tests.

Refer to Figure 6 for a summary of all the test bed components. 


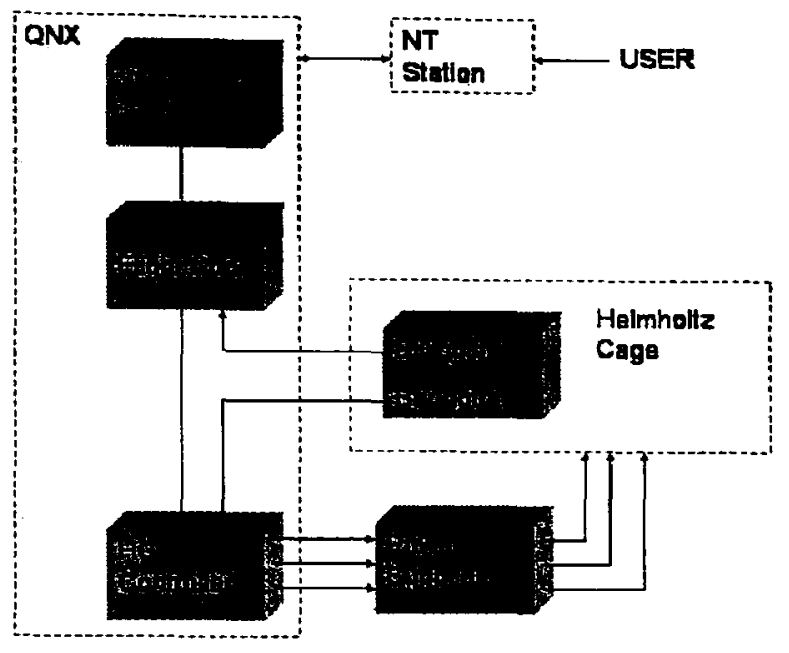

Figure 6: The Hardware in the loop System

\subsection{RACE Components}

RACE was made of several hardware and software components as well. There were two distinct computers, the user interface and the internal computer. Both systems run on Windows operating systems and had wireless communication available during experiments. The RACE sensors and actuators were controlled by the internal computer.

\subsubsection{RACE Sensors}

There were two sensors used on board RACE, an optical sensor and a rate sensor.

The optical sensor measured the angular position of RACE relative to the stationary base. The stationary disk on the base was split into 2048 sections, and the rotating device was able to determine the position by reading the sections on the disk. Hence, position could be determined to an accuracy of $0.175^{\circ}$. The angular velocity could also be determined using this base encoder by measuring the number of sections covered in a time step. 
The angular rate was also determined with the on-board rate sensor. The sensor has many features, such as the lack of hysteresis, low power consumption and light weight. The manufacturer has stated that it has a bias over a temperature range $\left(6 \mathrm{deg} / \mathrm{sec}_{\mathrm{P}-\mathrm{P} \text {,max }}\right)$, and a room temperature drift of $0.01^{\circ}$ which was taken into consideration in modeling and in application with the hardware.

\subsubsection{Actuators}

Attitude control was achieved with two different torquing systems onboard, a reaction wheel and an air thruster, which was used to emulate a magnetorquer or similar device. The reaction wheel was the main actuator, while the air thruster was used mainly for momentum dumping when the reaction wheel saturated, although it could also aid in small adjustments of orientation.

The reaction wheel, with an inertia of $I_{w}=0.00321 \mathrm{~kg}-\mathrm{m}^{2}$ (compared with a maximum platform inertia of $I_{B}=3.5 \mathrm{~kg}-\mathrm{m}^{2}$ ) could apply a peak stall torque of $2.259 \mathrm{~N}-\mathrm{m}$, and a continuous stall torque of $0.670 \mathrm{~N}-\mathrm{m}$. The air thruster could produce a maximum torque of $0.0025 \mathrm{~N}-\mathrm{m}$. 


\section{Modeling}

\subsection{QuickSat Modeling}

The model of the Quicksat system was in place and was undergoing validation when magnetometer testing began.

The QuickSat system was modeled by separating the mission into different modes, corresponding to the different stages after orbital insertion. Mode 10 corresponds to the de-tumble phase which occurs directly after the satellite is released from the launcher.

In order to exit Mode 10, the satellite must be rotating on all axes at a rate less than $1 \%$. Mode 20 is for coarse sun pointing which entails bringing the y-axis of the spacecraft to within $5^{\circ}$ of the sun. Mode 30 was the deployment of the solar arrays. During Mode 40 the momentum wheel begins to spin up. Orbit normal acquisition occurs in Mode 50, nadir acquisition in Mode 60 and finally Mode 70 is fine sun pointing.

During Mode 10, the de-tumble phase, the satellite utilizes the Earth's magnetic field to dissipate momentum and slow the satellite down to a controllable state. It is during this phase that the magnetic field relative to the magnetometer is changing at the quickest rate. Due to these qualities, a significant portion of the testing of the magnetometer occurred in Mode 10.

Throughout the simulation, however, regardless of the system mode, the same dynamics, kinematics, external torques etc. are applicable. The main difference between the modes is in the control algorithms, which are not discussed in this text.

\subsubsection{System Dynamics}

The rate of change taken relative to the rotating satellite body frame of total angular momentum, $\mathbf{h}$, is, 


$$
\frac{d \mathbf{h}}{d t}=\tau_{e x t}-\omega^{\times} \mathbf{h}
$$

where, $\boldsymbol{\omega}^{\mathbf{x}}$, is the skew symmetric matrix of the angular velocities, defined as

$$
\boldsymbol{\omega}^{\times}=\left[\begin{array}{ccc}
0 & -\omega_{3} & \omega_{2} \\
\omega_{3} & 0 & -\omega_{1} \\
-\omega_{2} & \omega_{1} & 0
\end{array}\right]
$$

where $\boldsymbol{\omega}$ is the angular velocity of the spacecraft. The external torques, $\tau_{e x t}$, take into consideration the disturbance torques, $\tau_{d i s t}$, and the torque from the magnetic thrusters, $\boldsymbol{\tau}_{\text {mag }}$.

$$
\tau_{e x t}=\tau_{\text {dist }}+\tau_{m a g}
$$

The angular velocity was calculated as the product of inverse of the inertia matrix, $\mathbf{I}$, and angular momentum

$$
\boldsymbol{\omega}=\mathbf{I}^{-1} \mathbf{h}
$$

\subsubsection{Spacecraft Kinematics}

The kinematics of the system were represented using Euler parameters, $q_{1}$ and $\mathbf{q}$. The parameters are defined in terms of the axis of rotation, $\hat{\mathbf{n}}$ and the angle of rotation, $\phi$,

$$
\begin{aligned}
& q_{1}=\cos \frac{\phi}{2} \\
& \mathbf{q}=\hat{\mathbf{n}} \sin \frac{\phi}{2}
\end{aligned}
$$

Therefore, the rotation of the spacecraft in terms of Euler Parameters is 


$$
\tilde{\mathbf{q}}=\left[\begin{array}{c}
\dot{q}_{1} \\
\dot{\mathbf{q}}
\end{array}\right]=\frac{1}{2}\left[\begin{array}{cc}
0 & -\boldsymbol{\omega}^{T} \\
\boldsymbol{\omega} & -\boldsymbol{\omega}^{\times}
\end{array}\right]\left[\begin{array}{c}
q_{1} \\
\mathbf{q}
\end{array}\right]
$$

The rotation matrix that defines the orientation of the satellite in the Earth Centered Inertial frame can be found from the quaternion,

$$
\mathbf{C}=\left(1-2 \mathbf{q}^{T} \mathbf{q}\right) \mathbf{I}_{3 \times 3}+2 \mathbf{q} \mathbf{q}^{T}-2 q_{1} \mathbf{q}^{\times}
$$

where the skew symmetric for the vector portion of the quaternion is equivalent to that defined above for angular velocity, and $\mathbf{I}_{3 \times 3}$ is the identity matrix.

$$
\mathbf{I}_{3 \times 3}=\left[\begin{array}{ccc}
1 & 0 & 0 \\
0 & 1 & 0 \\
0 & 0 & 1
\end{array}\right]
$$

\subsubsection{Actuators}

The two types of actuators onboard Quicksat are the magnetic torquers and the momentum wheel. The reaction torque, $\tau_{m a g}$, of the magnetic torquers is defined by

$$
\tau_{m a g}=\mathbf{m} \times \mathbf{B}
$$

where, $\mathbf{B}$, is the magnetic field acting on the spacecraft, and $\mathbf{m}$ is the dipole moment, which is a function of the current passing through the torquer. The amount of current is determined by the control system based on the desired result.

There are two components of the torque produced by the reaction wheel, the command torque and the wheel friction. The command torque is a function of the input command that was determined from test data. The friction is modeled as a symmetric viscous friction model for simplicity, even though testing has shown that it is slightly asymmetric. 


\subsubsection{Disturbance Torques}

There are several types of disturbance torques that can affect the attitude and orbit of the spacecraft. Included in the modeling of QuickSat were the Aerodynamic, Gravity Gradient and Solar torques.

Aerodynamic Torque The aerodynamic torque, $\tau_{a}$, acting on the spacecraft is caused by the upper atmosphere and is due to the offset between the center of pressure of the spacecraft and the center of mass [11].

$$
\tau_{a}=\mathbf{r}_{c p} \times \mathbf{F}_{a}
$$

where, $\mathbf{r}_{c p}$ is the center of pressure in the body coordinates and the aerodynamic force vector, $\mathrm{F}_{a}$, is

$$
\mathbf{F}_{a}=\left(\frac{1}{2}\right) \rho|V|^{2} S C_{D} \frac{\mathbf{V}}{|V|}
$$

where, $\rho$ is the atmospheric density $[27],|V|$ is the scalar value of the velocity, $C_{D}$, the drag coefficient, $S$ is the spacecraft projected area, and $\frac{\mathbf{V}}{|V|}$ a unit vector in the direction of the velocity of the spacecraft.

Gravity Gradient Torque The gravity gradient torque is a result of the differential attraction of the two sides (the "lower" and "upper" sides relative to the body the object is orbiting) of an orbiting object having unequal principal moments of inertia. This torque will tend to rotate the object to align its minimum inertia axis with the local vertical [11]. The torque caused by the gravity gradient for a near circular orbit is,

$$
\boldsymbol{\tau}_{G}=3 \omega_{0} \hat{\mathbf{r}} \times \mathbf{I} \cdot \hat{\mathbf{r}}
$$


where, $\hat{\mathbf{r}}$ is the nadir vector, a unit vector pointing from the planet to the spacecraft, $\omega_{0}$ is the orbital rate, and $\mathbf{I}$ is the moment of inertia of the craft.

Solar Radiation Torque Solar radiation pressure applies a force per unit area over the satellite in the anti-solar direction. Changes in all orbital elements are possible due to this solar radiation.

The solar torque, $\tau_{s}$, can be modeled as follows

$$
\tau_{s}=\mathbf{r} \times \mathbf{F}_{s}
$$

Here, $\mathbf{r}$ is the vector pointing from the body center of mass to the spacecraft optical center of pressure, and $\mathbf{F}_{s}$ is a function of spacecraft reflectivity, $\mathrm{K}$, the projected area normal to the sun, $A_{p s}$, and the solar pressure, $p_{s}$ which is considered to be a constant value.

$$
\mathrm{F}_{s}=(1+K) p_{s} A_{p s}
$$

As noted above, the solar torque is independent of the spacecraft position or velocity relative to the Earth, as long as the vehicle is in the sunlight. In low earth orbit, the aerodynamic and gravity gradients tend to be the primary disturbances acting on the spacecraft, although the solar torque was included for completeness.

\subsubsection{Orbital Mechanics}

The orbit was defined as a Kepler Orbit. The position and velocity of the satellite was described using six orbital elements, $\left\{a, e, i, \Omega, \omega, t_{p}\right\}$

The semi-major axis, $a$, and the eccentricity, $e$, describe the shape of an elliptical orbit, with $e=0$ corresponding to a circular orbit. 
The elements defining the orientation of the orbit are the inclination, $i$, the argument of the ascending node, $\Omega$, and the argument of the periapsis, $\omega$. The argument of the ascending node is defined as the angle from the $\mathrm{x}$-axis of the Earth fixed reference frame to the ascending node of the line of nodes. The line of nodes is the line created from the points of intersection of the satellite orbit and the equatorial plane. The argument of the periapsis is the angle between the line of nodes, originating at the ascending node, and the periapsis. Refer to Figure 7 for a visual description of the orbital parameters.

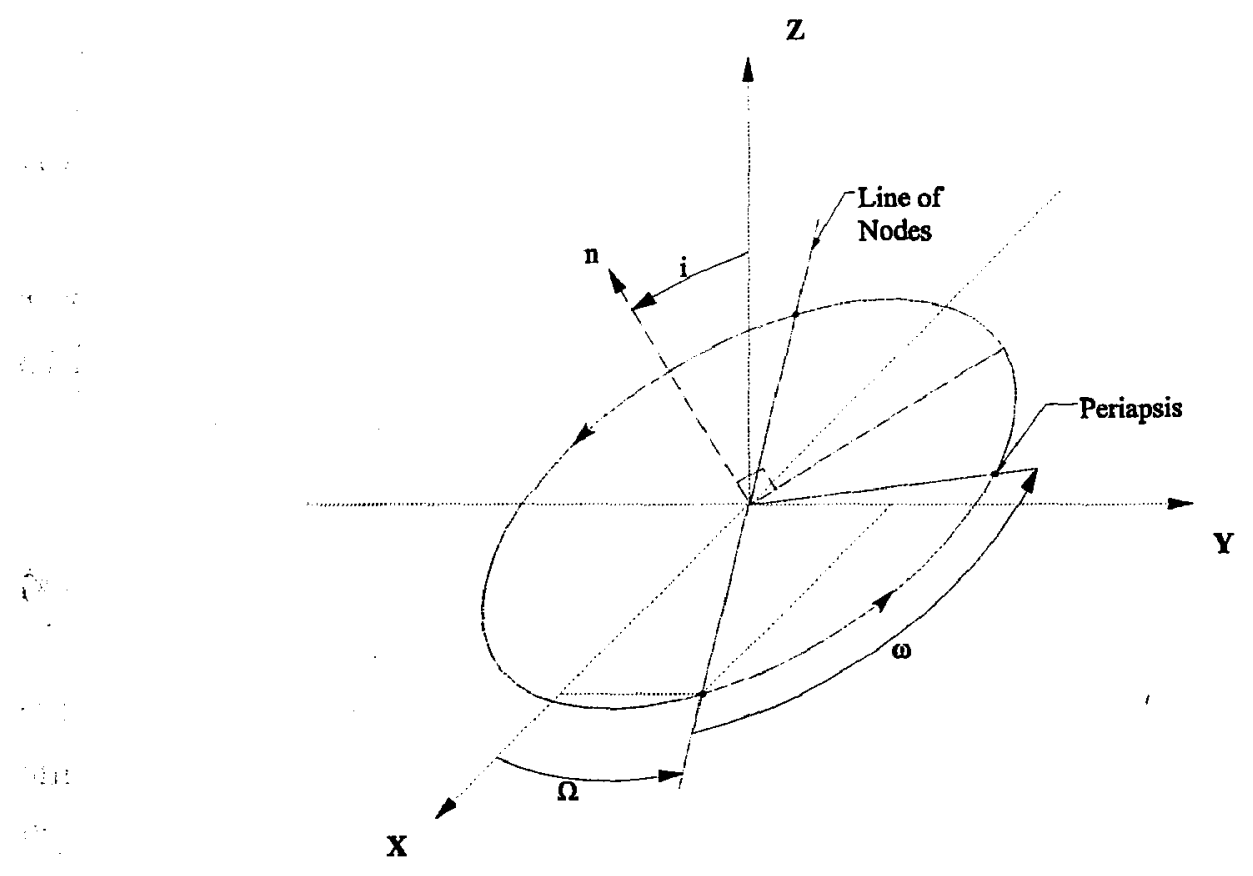

Figure 7: Definition of Orbital Parameters

The final of the orbital parameters, $t_{p}$, is the time of a relevant periapsis passing and is used to establish the epoch.

\subsubsection{Magnetic Field}

The magnetic field was represented with a spherical harmonic model using the coefficients from the International Geomagnetic Reference Field (IGRF). The Earth's magnetic field, B, 
is defined as the gradient of a scalar potential function, $V$, which is represented as a series of spherical harmonics.

$$
\begin{gathered}
\mathrm{B}=\nabla V \\
V(r, \theta, \phi)=a \sum_{n=1}^{k}\left(\frac{a}{r}\right)^{n+1} \sum_{m=0}^{n}\left(g_{n}^{m} \cos m \phi+h_{n}^{m} \sin m \phi\right) P_{n}^{m}(\theta)
\end{gathered}
$$

where a is the equatorial radius of the Earth, defined as $6371.2 \mathrm{~km}$ for the IGRF, $g_{n}^{m}$ and $h_{n}^{m}$ are Gaussian coefficients, determined from measurements of the Earth's magnetic field and $\mathrm{r}, \theta$, and $\phi$ are the distance from the Earth's centre, the coelevation, and the East Longitude from Greenwich; respectively. These last three variables can be combined to uniquely define any point in space relative to the Earth's centre. The first twelve empirically determined IGRF Gaussian coefficients from the 1995 data set were used in this model $(k=12)$.

The components of the magnetic field are

$$
\begin{gathered}
\mathbf{B}_{r}=-\frac{\partial V}{\partial r}=\sum_{n=1}^{k}\left(\frac{a}{r}\right)^{n+2}(n+1) \sum_{m=0}^{n}\left(g^{n, m} \cos m \phi+h^{n, m} \sin m \phi\right) P^{n, m}(\theta) \\
\mathbf{B}_{\theta}=-\frac{1}{r} \frac{\partial V}{\partial \theta}=-\sum_{n=1}^{k}\left(\frac{a}{r}\right)^{n+2} \sum_{m=0}^{n}\left(g^{n, m} \cos m \phi+h^{n, m} \sin m \phi\right) \frac{\partial P^{n, m}(\theta)}{\partial \theta} \\
\mathbf{B}_{\phi}=-\frac{1 \sin \theta}{r} \frac{\partial V}{\partial \phi}=-\frac{1}{\sin \theta} \sum_{n=1}^{k}\left(\frac{a}{r}\right)^{n+2} \sum_{m=0}^{n} m\left(-g^{n, m} \sin m \phi+h^{n, m} \cos m \phi\right) P^{n, m}(\theta)
\end{gathered}
$$

where, $g^{n, m}$ and $h^{n, m}$ are Schmidt normalized Gaussian coefficients, and $P^{n, m}$ are Schmidt normalized Legendre functions. 


$$
\begin{aligned}
& g^{n, m}=S_{n, m} g_{n}^{m} \\
& h^{n, m}=S_{n, m} h_{n}^{m} \\
& P_{n}^{m}=S_{n, m} P^{n, m}
\end{aligned}
$$

where the Schmidt Coefficient, $S_{n, m}$ is defined as

$$
S_{n, m} \equiv\left[\frac{\left(2-\delta_{m}^{o}\right)(n-m) !}{(n+m) !}\right]^{\frac{1}{2}} \frac{(2 n-1) ! !}{(n-m) !}
$$

The Kronecker delta function, $\delta$, is defined as

$$
\delta_{j}^{i}= \begin{cases}1 & i=j \\ 0 & i \neq j\end{cases}
$$

The reason the coefficients and functions in this Magnetic Field Model must be normalized in this fashion is because the model itself assumes that the Legendre polynomials are normalized such that

$$
\int_{0}^{\pi}\left[P_{n}^{m}(\theta)\right]^{2} \sin \theta \mathrm{d} \theta=\frac{2\left(2-\delta_{m}^{0}\right)}{2 n+1}
$$

Once the magnetic field is known in the $r, \theta, \phi$ frame of reference it can easily be transferred into any desired frame of reference for the model. 


\subsection{RACE Modeling}

System modeling of RACE was implemented in a MatLab Simulink model so that testing of the controller and the Kalman filter could be carried out before applying them to the hardware.

\subsubsection{Dynamics}

The dynamics of the RACE system for the rigid configuration can be viewed as a spinning platform plus wheel with the following equations of motion, where $I_{B}$ is the RACE moment of inertia without including the wheel and $I_{W}$ is the moment of inertia of the wheel.

$$
\left[\begin{array}{cc}
I_{B}+I_{W} & I_{W} \\
I_{W} & I_{W}
\end{array}\right]\left[\begin{array}{c}
\dot{\omega} \\
\dot{\omega}_{W}
\end{array}\right]=\left[\begin{array}{c}
g \\
g_{w}
\end{array}\right]
$$

$\omega$ is the angular rate of the RACE platform, and $\omega_{W}$ the angular rate of the wheel. $g$ is the net torque acting on the platform plus wheel and $g_{w}$ is the net torque acting on the wheel about its spin axis.

\subsubsection{State Space Model}

A state space model was developed to better analyse and design the Kalman filter and control law for RACE. It was decided that the state vector, $\mathbf{x}$, be composed of the angular position, $\theta$, the angular rate, $\omega$, and the rate sensor bias, $\beta$.

$$
\mathbf{x}=\left[\begin{array}{lll}
\theta & \omega & \beta
\end{array}\right]^{T}
$$

A state space model follows the form 


$$
\begin{aligned}
& \dot{\mathbf{x}}=\mathbf{F} \mathbf{x}+\mathbf{G u}+\mathbf{W} \eta \\
& \mathbf{y}=\mathbf{H} \mathbf{x}
\end{aligned}
$$

in terms of the system, $\mathbf{F}$, input, $\mathbf{G}$, measurement, $\mathbf{H}$ and system noise, $\mathbf{W}$, matrices.

The rate of change of the angular position is equal to the angular velocity, thus the system matrix is simply

$$
\mathbf{F}=\left[\begin{array}{lll}
0 & 1 & 0 \\
0 & 0 & 0 \\
0 & 0 & 0
\end{array}\right]
$$

To define the angular rate, Equation 24 was rearranged and the values of inertia $\left(I_{B}=\right.$ $3.5 \mathrm{~kg}-\mathrm{m}^{2}$ and $I_{w}=0.00321 \mathrm{~kg}-\mathrm{m}^{2}$ ) were included.

$$
\left[\begin{array}{c}
\dot{\omega} \\
\dot{\omega}_{W}
\end{array}\right]=\left[\begin{array}{cc}
0.5286 & -0.5286 \\
-0.5286 & 345.3562
\end{array}\right]\left[\begin{array}{c}
g \\
g_{w}
\end{array}\right]
$$

Therefore the rate of change of angular velocity of the platform is

$$
\dot{\omega}=0.5286 g-0.5286 g_{w}
$$

$1, \cdots$

where $g$ and $g_{w}$ are considered the inputs into the system, $\mathbf{u}=\left[\begin{array}{ll}g & g_{w}\end{array}\right]^{T}$. Therefore the feedback matrix, G, can be written in the form

$$
\mathbf{G}=\left[\begin{array}{cc}
0 & 0 \\
0.5286 & -0.5286 \\
0 & 0
\end{array}\right]
$$

The sensor bias was modeled as 


$$
\frac{d \beta}{d t}=\eta
$$

where $\eta$ is a white noise term.

This is the only term that contains a noise component, therefore the system noise matrix is

$$
\mathbf{W}=\left[\begin{array}{l}
0 \\
0 \\
1
\end{array}\right]
$$

The only two measurement instruments onboard RACE are the base encoder for angular position and the rate sensor for the angular rate. The output from the rate sensor is a combination of the sensor bias and the angular rate of RACE. Therefore the output matrix is

$$
\mathbf{H}=\left[\begin{array}{lll}
1 & 0 & 0 \\
0 & 1 & 1
\end{array}\right]
$$

Therefore assembling these definitions in a state space equation in terms the the state vector, $\mathbf{x}$, and the output vector, $\mathbf{y}$, yields

$$
\begin{aligned}
& \dot{\mathbf{x}}=\left[\begin{array}{lll}
0 & 1 & 0 \\
0 & 0 & 0 \\
0 & 0 & 0
\end{array}\right] \mathbf{x}+\left[\begin{array}{cc}
0 & 0 \\
0.5286 & -0.5286 \\
0 & 0
\end{array}\right] \mathbf{u}+\left[\begin{array}{l}
0 \\
0 \\
1
\end{array}\right] \eta \\
& \mathbf{y}=\left[\begin{array}{lll}
1 & 0 & 0 \\
0 & 1 & 1
\end{array}\right] \mathbf{x}
\end{aligned}
$$

However, when the Kalman filter was designed for RACE, the error in the state variable was used, which required changes to the standard state equations. This was done because the system the Kalman filter was designed for measures error in angular position directly. To 
facilitate the changes throughout the Kalman filter and state equation, the error in all terms was taken as a new state vector.

$$
\mathbf{e}=\mathbf{x}-\mathbf{x}_{\mathbf{r}}=\left[\begin{array}{c}
\theta \\
\boldsymbol{\omega} \\
\beta
\end{array}\right]-\left[\begin{array}{c}
\theta \\
\boldsymbol{\omega} \\
\beta
\end{array}\right]_{\text {desired }}
$$

where the "desired" sensor bias was taken to be zero.

Taking the derivative gives

$$
\dot{\mathbf{e}}=\dot{\mathbf{x}}-\dot{\mathbf{x}_{\mathbf{r}}}
$$

In terms of state, feedback, measurement, and system noise matrices, the system becomes

$$
\begin{aligned}
\dot{\mathbf{e}} & =\mathbf{F} \mathbf{x}+\mathbf{G u}+\mathbf{W} \eta-\mathbf{F} \mathbf{x}_{\mathbf{r}} \\
\dot{\mathbf{e}} & =\mathbf{F}\left(\mathbf{x}-\mathbf{x}_{\mathbf{r}}\right)+\mathbf{G u}+\mathbf{W} \eta
\end{aligned}
$$

Simplifying gives the final error state equation

$$
\dot{\mathbf{e}}=\mathrm{Fe}+\mathrm{Gu}+\mathrm{W} \eta
$$

And by defining the desired output vector as $\mathbf{y}_{r}=\mathbf{H x}_{r}$, the output equation and the final step in defining RACE in state space becomes.

$$
\mathbf{y}=\mathbf{H e}+\mathbf{y}_{\mathbf{r}}
$$




\subsubsection{Momentum Wheel}

The momentum wheel used in RACE was modeled to produce a linear output relative to the input with saturation limits of $\pm 6 \mathrm{~N} \mathrm{~m}$.

The friction in the momentum wheel was modeled as a coulomb plus viscous model which uses three parameters to define the model, $g_{o}, \omega_{o}$, and $b$. As the system friction profile was not determined experimentally, this friction model was chosen for simplicity. Refer to Figure 8 for a definition of the model parameters and Table 2 for the parameters used.

$$
g_{f r i c}(\omega)= \begin{cases}-g_{o}-b \omega & \omega>\omega_{o} \\ -\frac{g_{o}}{\omega_{o}} \omega-b \omega & -\omega_{o} \leq \omega \leq \omega_{o} \\ g_{o}-b \omega & \omega<\omega_{o}\end{cases}
$$

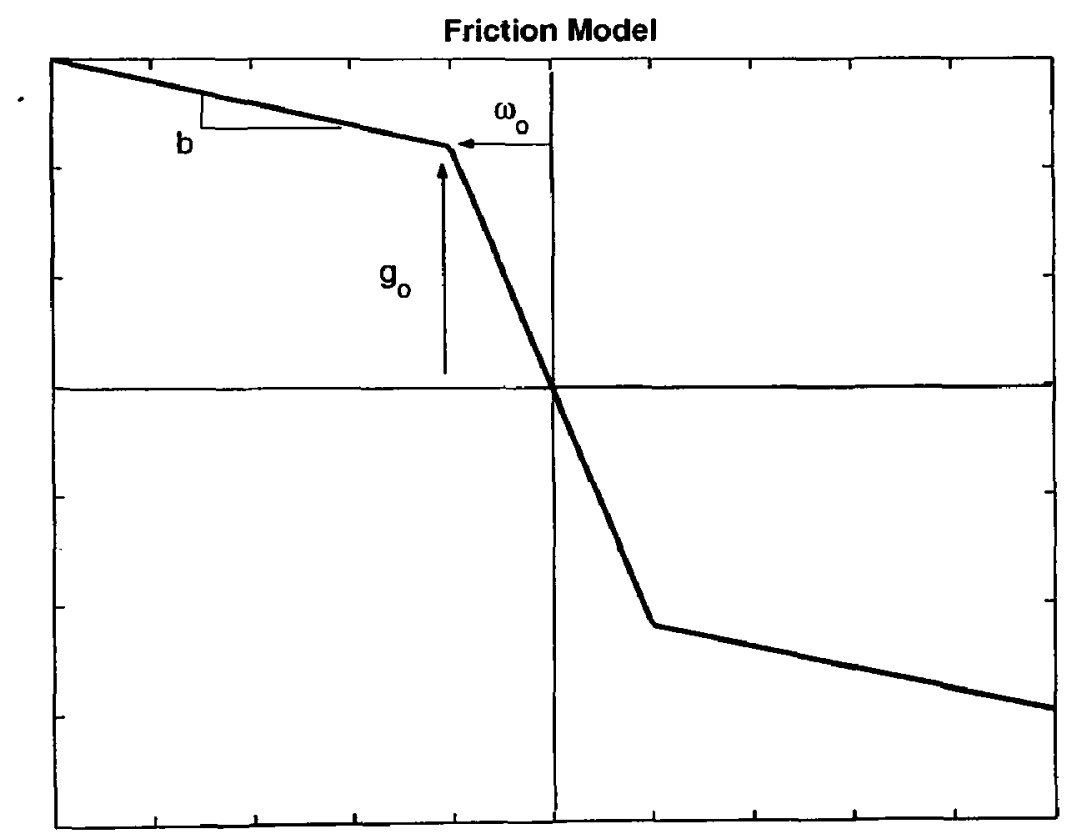

Figure 8: Friction Model

The coefficients used were chosen in simulation to slow the momentum wheel down to a stop 


\begin{tabular}{|c|c|}
\hline Coefficient & Value \\
\hline$g_{o}$ & $1.95 \mathrm{e}-5 \mathrm{Nm}$ \\
$\omega_{o}$ & $1 \mathrm{e}-6 \mathrm{rad} / \mathrm{s}$ \\
$b$ & $0.015 \mathrm{~kg} \cdot \mathrm{m}^{2} / s$ \\
\hline
\end{tabular}

Table 2: Friction Coefficients

in approximately five minutes from an initial spinning rate of $15 \%$ s. Refer to Figure 9 for the results of the friction model when applied to RACE.

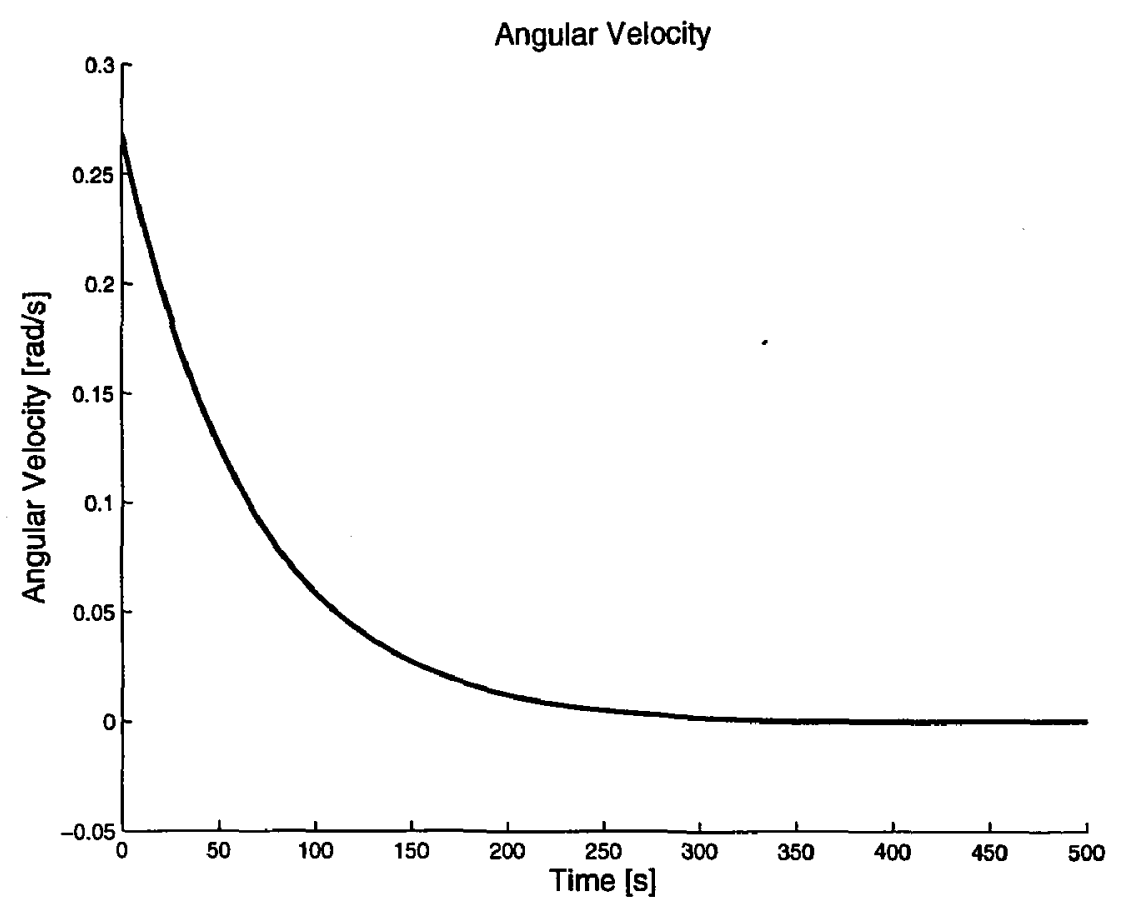

Figure 9: Angluar Velocity of RACE Momentum Wheel in the presence of friction only with initial rate of rotation of $15 \%$ 


\subsubsection{Rate Sensor}

A truth model of the rate sensor was included in the simulation. White noise was added to the measurement, as well as bias drift due to temperature variations, and the analog to digital output and gain to convert volts to radians per second were included for completeness. The modeling accuracy of this instrument was vital for testing the Kalman filter. 


\section{Controller Design for QuickSat Project}

There were three different tests that were carried out using hardware in the loop simulations with the Helmholtz coils. The first was to test the Helmholtz cage to verify that it was creating the necessary magnetic fields, the second was to test the flight qualified Billingsly magnetometer and the third was to test the control algorithm in the flight code in conjunction with the magnetometer. This required two controllers, one for the Helmholtz cage and one for the flight code.

A robust controller that was designed to track second order reference signals was used to control the coils, and a B-dot controller was chosen for the QuickSat simulation.

\subsection{Robust Controller}

The Helmholtz Cage was modeled under the assumption that the three coil axes behaved independently of each other and the external electronic disturbances were not accounted for. These two assumptions introduced uncertainty into the Helmholtz Cage model.

Therefore, a robust controller was chosen for each axis of the Helmholtz cage because of its ability to maintain stability in linear systems in the presence of uncertainties.

The robust controller that was chosen had been designed in an error space using a weighted error as the state. Within this error space, a feedback controller is used to drive the weighted error to zero in the presence of a non-decaying second order reference signal [8]. As it is a weighted error, small perturbations are quickly suppressed and the system remains stable.

The following is a synopsis of the development of the controller that was implemented on the Helmholtz coils. The generic controller was designed by Gene Franklin; David Powell and Abbas Emani-Naeini as outlined in their text book Control for Dynamic Systems [8]. The system under consideration is represented in state space in terms of the state vector $\mathbf{x}$, the input vector, $\mathbf{u}$ the noise vector $\eta$ and the output vector, $\mathbf{y}$. 


$$
\begin{aligned}
& \dot{\mathbf{x}}=\mathbf{F x}+\mathbf{G u}+\mathbf{W} \eta \\
& \mathbf{y}=\mathbf{H} \mathbf{x}
\end{aligned}
$$

where $\mathbf{F}$ is the system matrix, $\mathbf{G}$ the input matrix, $\mathbf{H}$ the measurement matrix and $\mathbf{W}$ the system noise matrix.

This controller was designed to follow a non-decaying reference signal, $\mathbf{r}$, of the form

$$
\ddot{\mathbf{r}}+\alpha_{1} \dot{\mathbf{r}}+\alpha_{2} \mathbf{r}=0
$$

Where, $\alpha_{1}$ and $\alpha_{2}$ were the coefficients that describe the path that the system must follow. The following is the development of the generic form of the controller. The error in the system is defined in terms of the output as

$$
\mathbf{e} \triangleq \mathbf{y}-\mathbf{r}
$$

By replacing the reference signal with the error signal and output signals, the system definition becomes

$$
\ddot{\mathbf{e}}+\alpha_{1} \dot{\mathbf{e}}+\alpha_{2} \mathbf{e}=\ddot{\mathbf{y}}+\alpha_{1} \dot{\mathbf{y}}+\alpha_{2} \mathbf{y}
$$

or in terms of the state vector

$$
\ddot{\mathbf{e}}+\alpha_{1} \dot{\mathbf{e}}+\alpha_{2} \mathbf{e}=\mathbf{H} \ddot{\mathbf{x}}+\alpha_{1} \mathbf{H} \dot{\mathbf{x}}+\alpha_{2} \mathbf{H} \mathbf{x}
$$

A new state vector, $\boldsymbol{\xi}$, represents the states in the error space, and is given in terms of the actual state vector as

$$
\boldsymbol{\xi} \triangleq \ddot{\mathbf{x}}+\alpha_{1} \dot{\mathbf{x}}+\alpha_{2} \mathbf{x}
$$


with a control input vector, $\mu$, in error space

$$
\boldsymbol{\mu} \triangleq \ddot{\mathbf{u}}+\alpha_{1} \dot{\mathbf{u}}+\alpha_{2} \mathbf{u}
$$

Combining the above system definition and state vector in error space, the system under consideration becomes

$$
\ddot{\mathrm{e}}+\alpha_{1} \dot{\mathrm{e}}+\alpha_{2} \mathbf{e}=\mathbf{H} \boldsymbol{\xi}
$$

The state equation in the error space is then defined as

$$
\dot{\boldsymbol{\xi}}=\dddot{\mathbf{x}}+\alpha_{1} \ddot{\mathbf{x}}+\alpha_{2} \dot{\mathbf{x}}=\mathbf{F} \boldsymbol{\xi}+\mathbf{G} \boldsymbol{\mu}
$$

A new variable, $\mathbf{z}=\left[\begin{array}{ccc}\mathbf{e} & \dot{\mathbf{e}} & \boldsymbol{\xi}^{T}\end{array}\right]^{T}$, is used to define the error state space system.

$$
\dot{\mathbf{z}}=\mathbf{A z}+\mathbf{B} \boldsymbol{\mu}
$$

where

$$
\mathbf{A}=\left[\begin{array}{ccc}
0 & 1 & \mathbf{0} \\
-\alpha_{2} & -\alpha_{1} & \mathbf{H} \\
\mathbf{0} & \mathbf{0} & \mathbf{F}
\end{array}\right] \quad \mathbf{B}=\left[\begin{array}{l}
\mathbf{0} \\
\mathbf{0} \\
\mathbf{G}
\end{array}\right]
$$

Applying a simple state feedback control law in the error space results in a controllable system in terms of gains, $\mathbf{K}$.

$$
\boldsymbol{\mu}=-\left[\begin{array}{lll}
K_{2} & K_{1} & \mathbf{K}_{\mathbf{0}}
\end{array}\right]\left[\begin{array}{c}
\mathbf{e} \\
\dot{\mathbf{e}} \\
\xi
\end{array}\right]=-\mathbf{K z}
$$


Translating this control law back into terms of the system states results in the following robust control law.

$$
\frac{d^{2}}{d t^{2}}\left(\mathbf{u}+\mathbf{K}_{\mathbf{0}} \mathbf{x}\right)+\sum_{i=1}^{2} \frac{d^{2-i}}{d t^{2-i}}\left(\mathbf{u}+\mathbf{K}_{\mathbf{0}} \mathbf{x}\right)=-\sum_{i=1}^{2}-K_{i} \frac{d^{2-i}}{d t^{2-i}}(\mathbf{e})
$$

In order to apply this controller to the Helmholtz cage, each axis was modeled individually. Along each axis, the rate of change of magnetic field is a function of the input, in this case the electric current in the wires being applied to the system and the response capabilities of the coils (discussed in Section 5.3); therefore, the state space system collapses to the following equations,

$$
\begin{aligned}
& \dot{x}=-a x+u \\
& y=x
\end{aligned}
$$

Where the state variable, $x$, was the magnetic field produced by each axis, and $a$ was the inverse of the time constant for the axis (refer to Section 5.3 for more information regarding the time constant) and $u$ was the input that will be determined by the controller.

In the case of the Helmholtz Cage, each axis followed the commanded magnetic field controlled by the input current in the coils. A sine wave was used as the reference signal to design the controller. It was assumed that the magnetic field as felt by the magnetometer in orbit would be oscillating and periodic, therefore the Helmholtz cage would need to create a similar field.

$$
r=\sin \omega
$$

Taking the second derivative

$$
\ddot{r}=-\omega^{2} \sin \omega
$$


Therefore, a sine wave can be written in the differential form as

$$
\ddot{r}+\omega^{2} r=0
$$

where $\omega$ is the frequency of the sine wave and $\alpha_{1}=0$ and $\alpha_{2}=\omega^{2}$.

Referring to Equations 41 and 53, it can be noted that $\mathbf{H}=[a], \mathbf{F}=[1]$ and $\mathbf{G}=[1]$. Therefore each axis of the Helmholtz Cage is defined in this error space as (Equation 49)

$$
\dot{\mathbf{z}}=\left[\begin{array}{ccc}
0 & 1 & 0 \\
-\omega^{2} & 0 & a \\
0 & 0 & 1
\end{array}\right] \mathbf{z}+\left[\begin{array}{l}
0 \\
0 \\
1
\end{array}\right] \boldsymbol{\mu}
$$

From this error space definition, the controller (Equation 52) for the Helmholtz cage can be written as

$$
\ddot{\mathbf{u}}+\mathbf{K}_{0} \ddot{\mathbf{x}}+\omega^{2}\left(\mathbf{u}+\mathbf{K}_{0} \mathbf{x}\right)=-K_{1} \dot{\mathbf{e}}-K_{2} \mathbf{e}
$$

for each axis of the Helmholtz Cage. The robust controller for the Helmholtz Cage axes is summarized in Figure 10

\subsection{B-dot Control Law}

A B-dot controller is a common attitude control method on satellites. This type of controller was implemented into the QuickSat simulation and will become part of the satellite flight software. B-dot control is used to stabilize the satellite after separation from the launching system by using the measured magnetic field as rate feedback.

This control law will help to dissipate the kinetic energy, $E$, present during free tumbling and bring the spacecraft into a controllable state. 


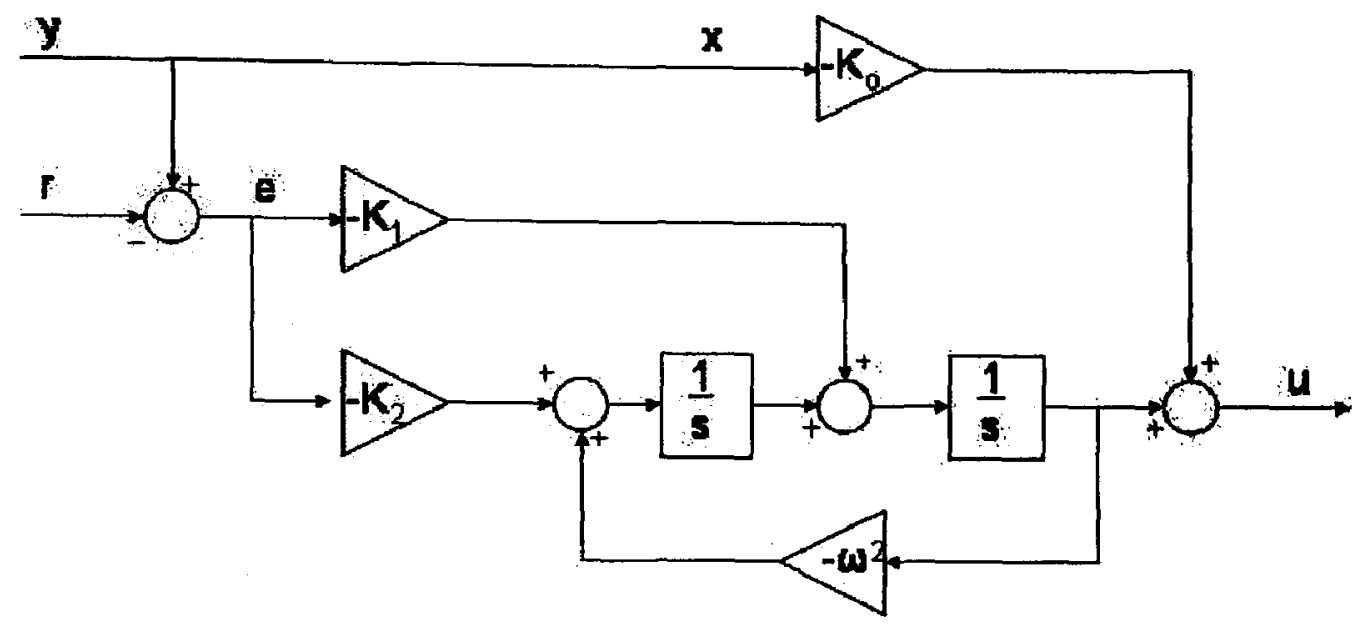

Figure 10: Robust Controller Block Diagram

The rate of change of kinetic energy of the system in the body-centered frame was

$$
\dot{E}=\omega \cdot \tau_{m a g}
$$

In this case, $\omega$ was the angular velocity of the satellite and the torque, $\tau_{\text {mag }}$, was provided by the magnetic torquers and was defined as 


$$
\tau_{\text {mag }}=\mathbf{m} \times \mathbf{B}
$$

where $\mathbf{m}$ was the net internal magnetic moment and $\mathbf{B}$ was the Earth's magnetic field. Combining these two and utilizing the identity $a \cdot(b \times c)=c \cdot(a \times b)$, the above could be rewritten as

$$
\begin{aligned}
& \dot{E}=\boldsymbol{\omega} \cdot(\mathbf{m} \times \mathbf{B}) \\
& \dot{E}=-\boldsymbol{\omega} \cdot(\mathbf{B} \times \mathbf{m}) \\
& \dot{E}=-\mathbf{m} \cdot(\boldsymbol{\omega} \times \mathbf{B})
\end{aligned}
$$

The dipole moment was chosen in terms of gain, $\mathbf{K}$, i.e.,

$$
\mathbf{m}=\mathbf{K}(\boldsymbol{\omega} \times \mathbf{B})
$$

Thus the kinetic energy equation became

$$
\dot{E}=-(\boldsymbol{\omega} \times \mathbf{B}) \cdot \mathbf{K}(\boldsymbol{\omega} \times \mathbf{B})
$$

The result of this equation will be negative so long as $\mathbf{K}$ is non-negative definite, which means there will be a continual energy loss.

When a spacecraft is tumbling, the change in magnetic field, observed by the sensors onboard, is primarily due to the rotation of the spacecraft, rather than its position in the orbit. The observed rate of change with respect to the rotating satellite frame of magnetic field could be written as

$$
\dot{\mathrm{B}} \approx-\boldsymbol{\omega} \times \mathrm{B}
$$

Therefore, the magnetic moment that will be applied by the control law is 


$$
\mathbf{m}=-\mathbf{K} \dot{\mathbf{B}}
$$




\section{Testing of Helmholtz Cage}

Several tests were done on the Helmholtz cage to determine characteristics and properties of the system in order to select appropriate parameters for the Helmholtz cage controller.

First, the magnetometer calibration factors were tested by comparing the magnetometer output values against each other and a teslameter. Then the cage itself was characterized, determining how each axis behaved with respect to input current and under the influence of each other. Finally, the transfer function for each axis was determined experimentally.

\subsection{Verification of Magnetometer Measurements}

The first step was to confirm the magnetometer calibration factors specified by the manufacturer. Values of the Earth's magnetic field read from a teslameter were compared to the values determined by the magnetometers.

Several static measurements were taken along the axes of the Barington and Billingsley magnetometers, the averages from the Barington magnetomter and the teslameter can be found in Table 3 .

\begin{tabular}{|c|c|c|c|}
\hline Axis & Static Magnetic Field [G] & Barington Measurements [G] & Percent Error [\%] \\
\hline X-Axis & 0.1192 & 0.1100 & 7 \\
Y-Axis & -0.125 & -0.123 & 1.6 \\
Z-Axis & -0.4561 & 0.4540 & $<1$ \\
\hline
\end{tabular}

Table 3: Static Magnetic Field Measurements At Center of Helmholtz Cage

The Barington magnetometer was found to read within $10 \%$ of the Earth's magnetic field on all axes. It was concluded that the calibration factor given in the users manual $(10 \mathrm{~V} / \mathrm{G})$ was acceptable.

However, the Billinglsy manufacturer specified calibration factor did not show consistent 
values with the teslameter and the Barington instrument. Therefore, a calibration factor was experimentally determined to be $15.84 \mathrm{~V} / \mathrm{G}$.

\begin{tabular}{|c|c|c|c|}
\hline Axis & Magnetic Field [G] & Billingsley Measurements [V] & Scaling Factor [V/G] \\
\hline X-Axis & 0.1192 & 1.79 & 15.01 \\
Y-Axis & -0.125 & -1.94 & 15.52 \\
Z-Axis & -0.4561 & -7.75 & 16.99 \\
\hline
\end{tabular}

Table 4: Billingsley Scaling Factor

\subsection{Axis Linearity and Coupling}

It was necessary to determine the linearity between the input current and the output magnetic field (in terms of voltage). This same test was used to determine whether the three axes behaved independently of each other.

The current along each axis was increased incrementally, while the output voltage for each of the three axes was observed. The test was repeated while decreasing current as well to determine if there was a hysteresis effect in the system. This method tested only the static measurements of the coils, and was not dependent on dynamic capabilities of the coils. Note that these tests need to be repeated if the coils are moved as proper functioning of the coil system is dependent on the local magnetic field.

Refer to Figures 11 to 13 for the results of each axis.

A linear least-squares regression and an $R^{2}$ value was calculated for each axis to determine the correlating factor between the current and voltage or the coil output gain. $R^{2}$ is defined as the statistical measure of how well a regression line approximates real data points; an $R^{2}$ value of $1(100 \%)$ indicates a perfect fit. 


$$
R^{2}=\frac{1-\sum_{i=1}^{n}\left(y_{i}-\hat{y}_{i}\right)^{2}}{\sum_{i=1}^{n} y_{i}^{2}-\left[\frac{\sum_{i=1}^{n} y_{i}^{2}}{n}\right]}
$$

where $y_{i}$ is the data point and $\hat{y}_{i}$ is the regression line data point.

Refer to Table 5 for the numerical results of the gains found during these tests.

\begin{tabular}{|c|c|c|}
\hline \multicolumn{3}{|c|}{ Input Current in X-Axis } \\
\hline Output Axis & Coil Gain [V/A] & $R^{2}$ \\
\hline X & 13.198 & 1 \\
Y & -0.5373 & 0.9929 \\
Z & 0.0521 & 0.5943 \\
\hline \multicolumn{3}{|c|}{ Input Current in Y-Axis } \\
\hline X & 0.5803 & 0.9982 \\
Y & 12.306 & 0.9998 \\
Z & -0.0169 & 0.1003 \\
\hline \multicolumn{3}{|c|}{ Input Current in Z-Axis } \\
\hline X & -0.1214 & 0.9787 \\
Y & 0.0486 & 0.4899 \\
Z & 12.696 & 1 \\
\hline
\end{tabular}

Table 5: Values of Coil Gains and Their Associated $R^{2}$ Values

The axis linearity tests showed that the linear relationship between output and input was stable and consistent, and no hysteresis was present in the system, which made the coils easy to control with reliable results. However, it also demonstrated that the coils do not behave entirely independently. Figure 11 and Figure 12 demonstrate that the X and Y Axes are coupled. 


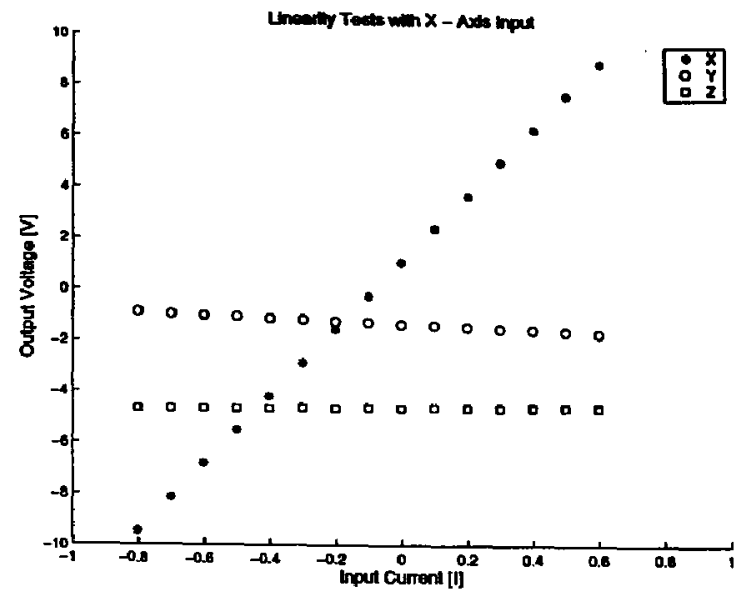

Figure 11: Relationship between Input Current Along X-Axis and Output Voltage of Magnetometer

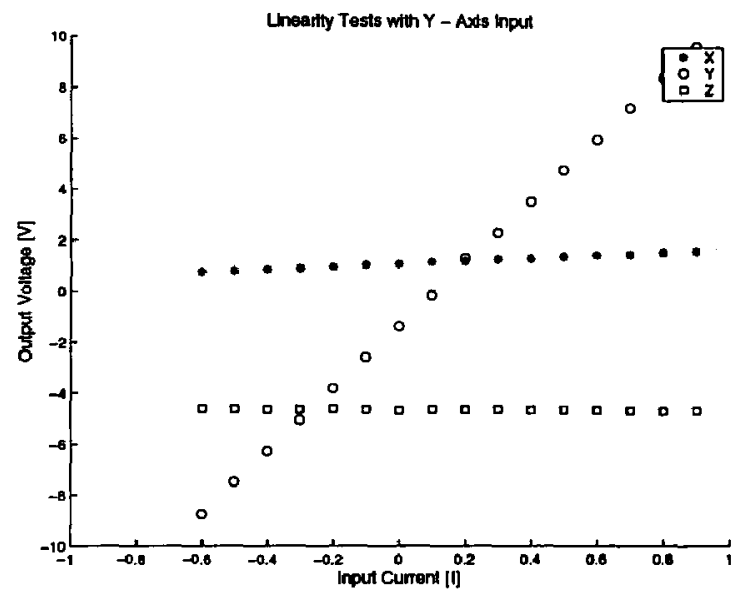

Figure 12: Relationship between Input Current Along Y-Axis and Output Voltage of Magnetometer

The gains were used to construct a driving matrix, Equation 67, which was used for the open loop control of the coils. This matrix took the inverse of the relationship between input current and output voltage (equivalently magnetic field), so that given the desired magnetic field in Gauss and the appropriate current in Amperes to achieve the necessary 


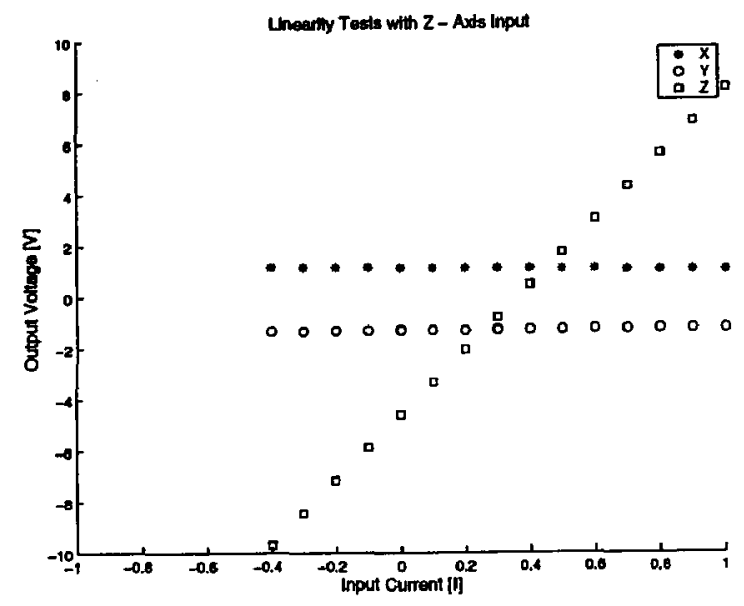

Figure 13: Relationship between Input Current Along Z-Axis and Output Voltage of Magnetometer

field could be calculated.

$$
\left[\begin{array}{c}
i_{X} \\
i_{Y} \\
i_{Z}
\end{array}\right]=\left[\begin{array}{ccc}
0.7562 & 0.0330 & 0 \\
-0.0356 & 0.8111 & 0 \\
0 & 0 & 0.7876
\end{array}\right]\left[\begin{array}{c}
B_{d e s, X} \\
B_{d e s, Y} \\
B_{d e s, Z}
\end{array}\right]-\left[\begin{array}{c}
0.0820 \\
-0.1092 \\
-0.3611
\end{array}\right]
$$

To check the validity of this driving matrix, it was implemented in the hardware in the loop simulation as an open loop controller. A step input was applied to each axis to view the response of all three axes, and through multiple tests, it was determined that though the axes appeared to be coupled, they behaved best when they were controlled independently, and thus the driving matrix was reduced to a diagonal matrix.

With such a controller all three axes responded with a maximum of $5 \%$ steady state error, and with a consistent 0.25 second delay. As the simulation was run at $4 \mathrm{~Hz}$, which is the maximum capability of the hardware/software combination, it is valid to assume that this apparent delay is software based, rather than a limitation in the Helmholtz cage itself. Refer to Figure 14 to Figure 16 for the step response of each axis. 


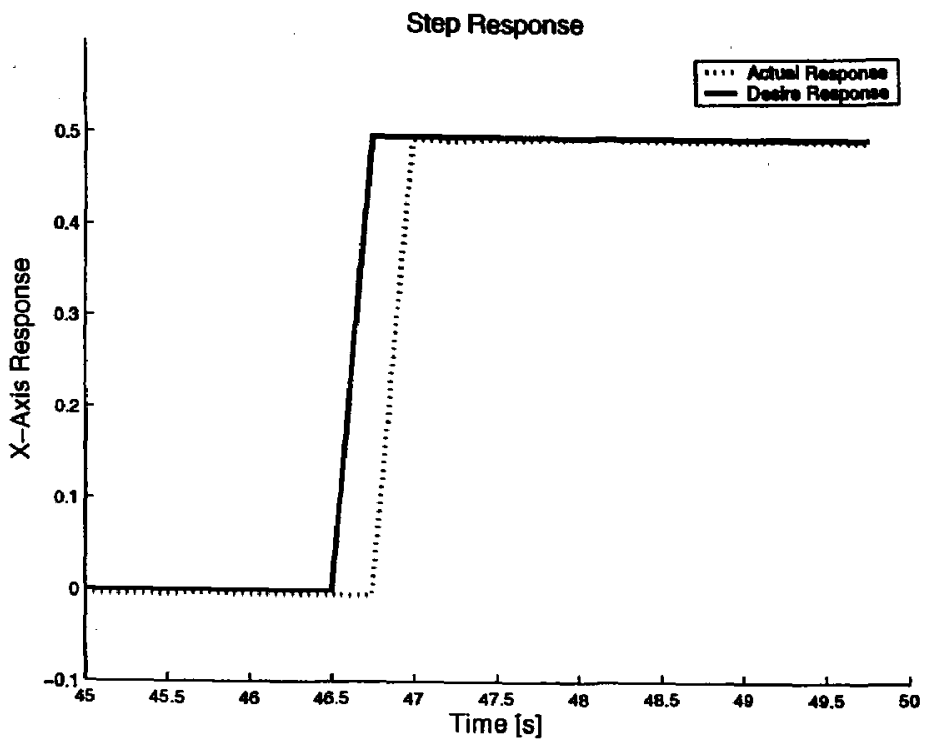

Figure 14: Response Along the X-Axis to a Step Input

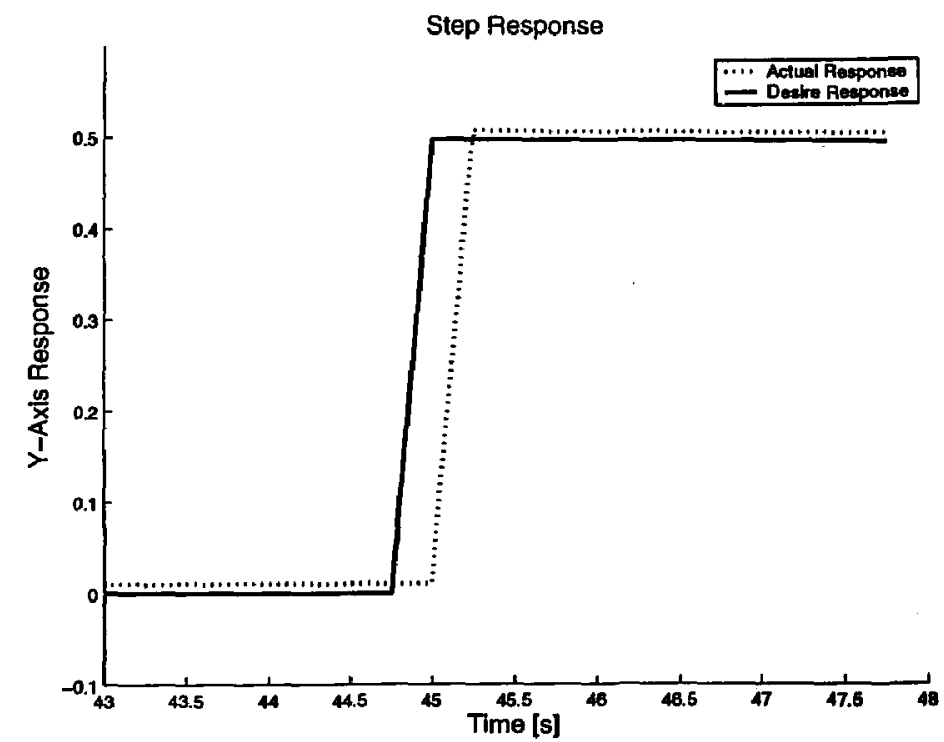

Figure 15: Response Along the Y-Axis to a Step Input 


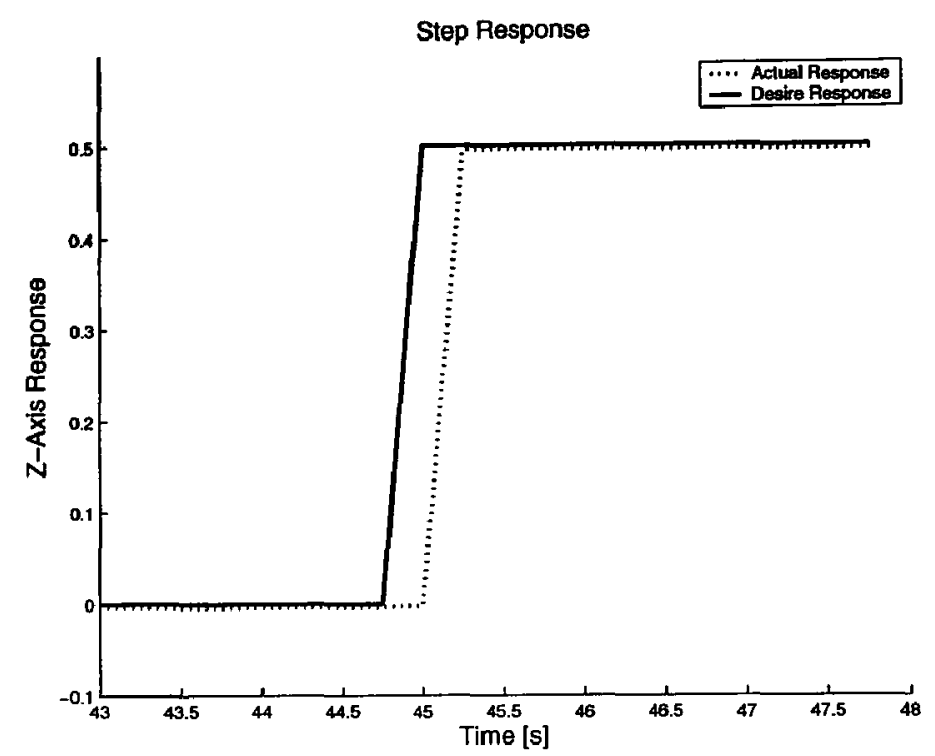

Figure 16: Response Along the Z-Axis to a Step Input

\subsection{Transfer Function}

Determining the transfer function of the coils is important in order to be able to determine the proper parameters for the controller. Based on the results from Section 5.2 it was decided to apply a control law for each axis individually. Therefore, the transfer function was determined experimentally for each axis independently.

Each axis of the Helmholtz cage obeys the following

$$
V=L \frac{d i}{d t}
$$

where $i$ is input current, $V$ is output voltage and the coils themselves behave as the inductor, $L$. Given that this is a first order equation, the transfer function for this first-order system can be written as

$$
G(s)=\frac{1}{s+a}
$$


where $a$ is the inverse of the time constant, $a=\frac{1}{\tau}$. To determine the time constant, which is the time equivalent for the response to reach $67.3 \%$ of its final value, the circuit in Figure 17 was constructed. A resistor of value $100 \Omega$ was added in series with the axis of the Helmholtz cage under consideration. A square waveform (equivalent to a step input) was introduced into the system and the voltage measured across the coils was analyzed on an oscilloscope. The time constants of each axis can be found in Table 6 .

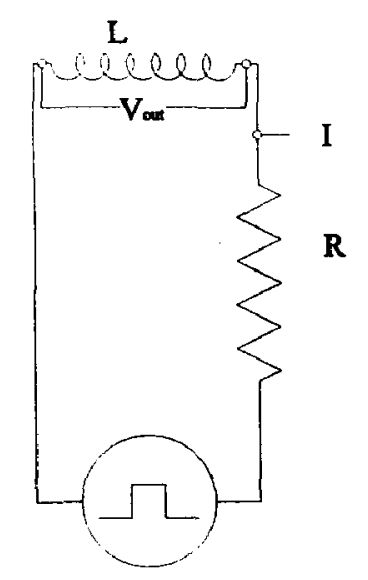

Figure 17: Circuit for determining the Time Constant of the coils

\begin{tabular}{|c|c|}
\hline Axis & Time Constant $[\mu \mathrm{s}]$ \\
\hline $\mathrm{X}-$ Axis & 0.1589 \\
Y - Axis & 0.1572 \\
$\mathrm{Z}-$ Axis & 0.1605 \\
\hline
\end{tabular}

Table 6: Time Constants for the Coil System 


\section{QuickSat Hardware in the Loop Simulation}

Two types of tests were carried out with the QuickSat system. There were those that were for testing the Helmholtz Cage itself, to verify its ability to test the magnetometer. The second type of hardware in the loop simulations was to test the flight qualified magnetometer.

\subsection{Helmholtz Cage Controller Implementation}

Application of a robust controller to the Helmholtz cage was a straightforward process. Each axis was controlled separately and thus three controllers were designed, all having the same structure described in Section 4.1.

It was determined in simulation that each axis must be able to create a field that could change at a maximum of $6.3 \% / \mathrm{s}(=0.1 \mathrm{rad} / \mathrm{s})$ and so the frequency of the sine wave chosen for the reference'signal was $\omega=0.1 \mathrm{rad} / \mathrm{s}$.

The gains of the controller were chosen initially using pole placement and were fine tuned in simulation.

The gains were chosen in an effort to limit steady state error, as well as minimize the settling time. Table 7 shows the robust controller gains chosen for each axis.

\begin{tabular}{|c|c|c|c|}
\hline$\backslash$ & X-Axis & Y-Axis & Z-Axis \\
\hline $\mathrm{K}_{o}$ & -1.7933 & -1.7305 & -1.7305 \\
$\mathrm{~K}_{1}$ & 1.0313 & 1.0416 & 1.0416 \\
$\mathrm{~K}_{2}$ & 0.4695 & 0.4743 & 0.4743 \\
\hline
\end{tabular}

Table 7: Robust Controller Gains for Helmholtz Cage

Once the two magnetometers were in place, a hardware in the loop test was run to test the abilities of the system - both the ability of the coils to create the desired field and the ability of the Billingsly magnetometer to follow the created field. 
Refer to Figures 18 and 19 for the results of the Helmholtz Cage controller with the sine wave input.

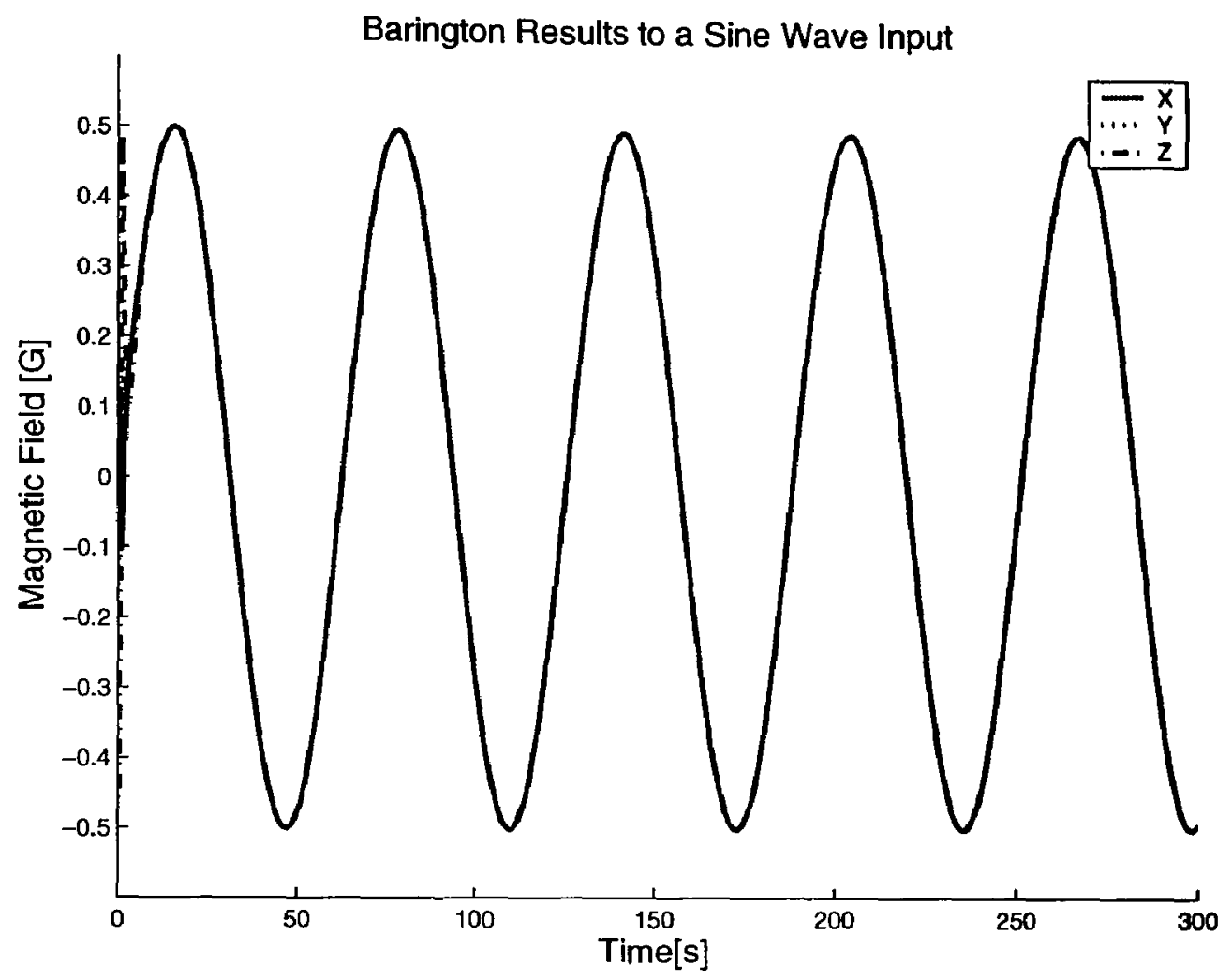

Figure 18: Response of the Coils to Sine Wave Input, Measured with the Barington Magnetometer

All three axes responded well to the controller.

\subsubsection{Misalignment of Magnetometers}

As it can be noted from Figure 19, the results found from the Billingsly magnetometer were not identical to those found from the Barington Magnetometer. Initially it was assumed that the axes of the two magnetometers were not properly aligned. To minimize the error 


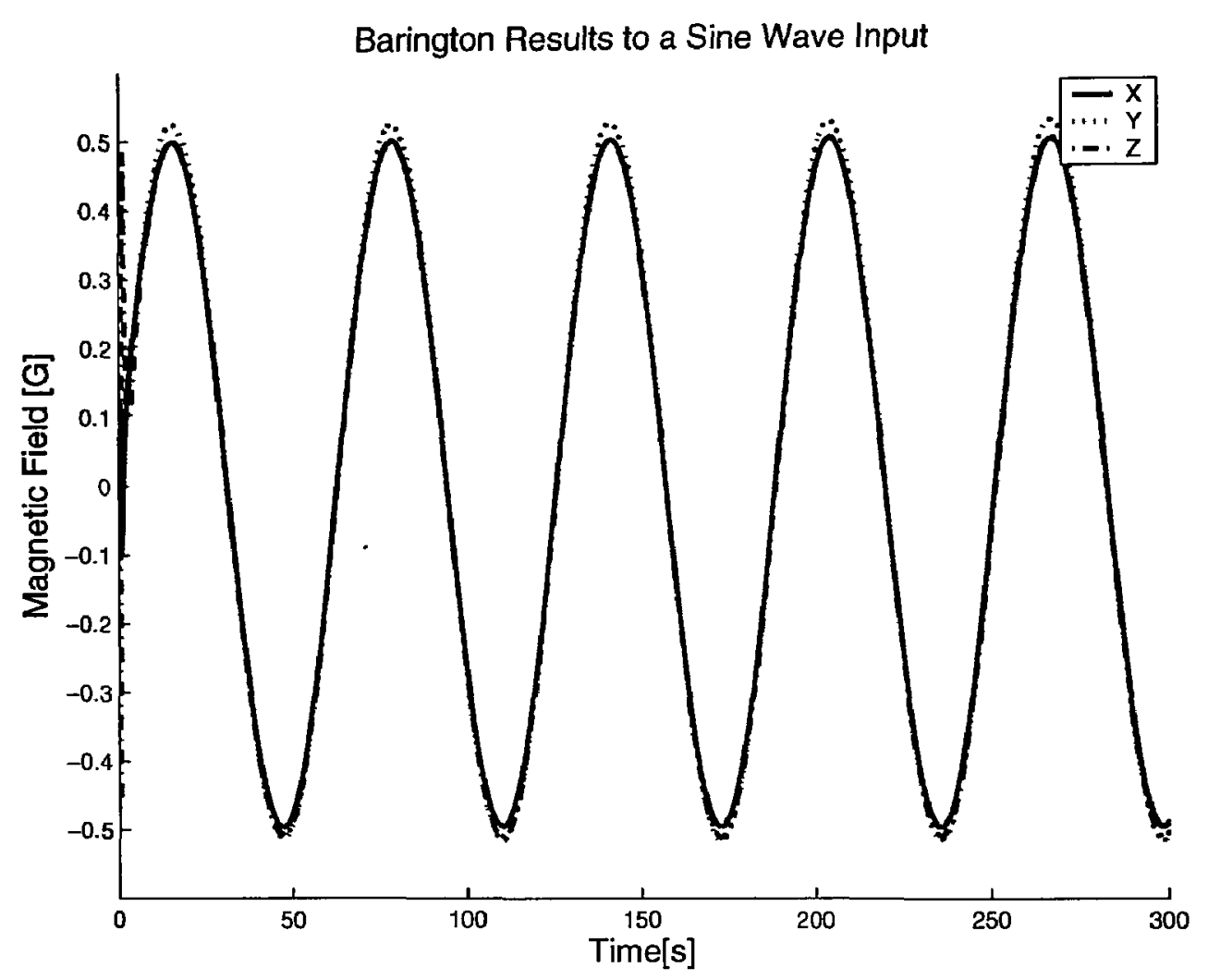

Figure 19: Response of the Coils to sine Wave Input, Measured with the Billingsly Magnetometer 
caused by this perceived misalignment, the data was to be corrected once the simulation was completed by applying a rotation to the Billinglsy data.

However, implementation of this rotation matrix was only able to improve the results found on one axis at a time and any compromise of the three axes reduced the quality of results overall.

The magnitude of the field was then calculated and plotted in Figure 20 which indicates that the difference between the two measurements was in fact not due to a misalignment, as the two systems would have shown the same magnitude if misalignment was the source of the error. The error was attributed to a combination of the noise in the system and the approximation of the previously determined gain for the Billingsly magenetometer, as it was a compromise between all three axes. Finally, no correction factors were applied to the results of either magnetometer for analysis.

\subsection{Hardware in the Loop with QuickSat Simulation}

As stated in Section 3.1, the greatest rate of change of magnetic field as felt by the magnetometer will occur during the de-tumbling phase of the mission and hence the majority of the testing was carried out during this phase alone.

\subsubsection{Open Loop Simulation}

During the first phase of the Billingsly validation tests, the magnetometer was merely reading values, but was not inputting them back into the QuickSat Simulation. For a first pass, initial conditions were arbitrarily chosen to have a roll, pitch and yaw velocity of $2.0,1.5$, and $0.5 \% \mathrm{~s}$ respectively.

Figure 21 demonstrates the ability of the Billingsly Magnetometer to determine the magnetic field of a satellite in orbit. Furthermore, as the Helmholtz cage is capable of creating the anticipated magnetic field (refer to Figure 22), the assumption of using a sine wave to develop the controller proves valid. 


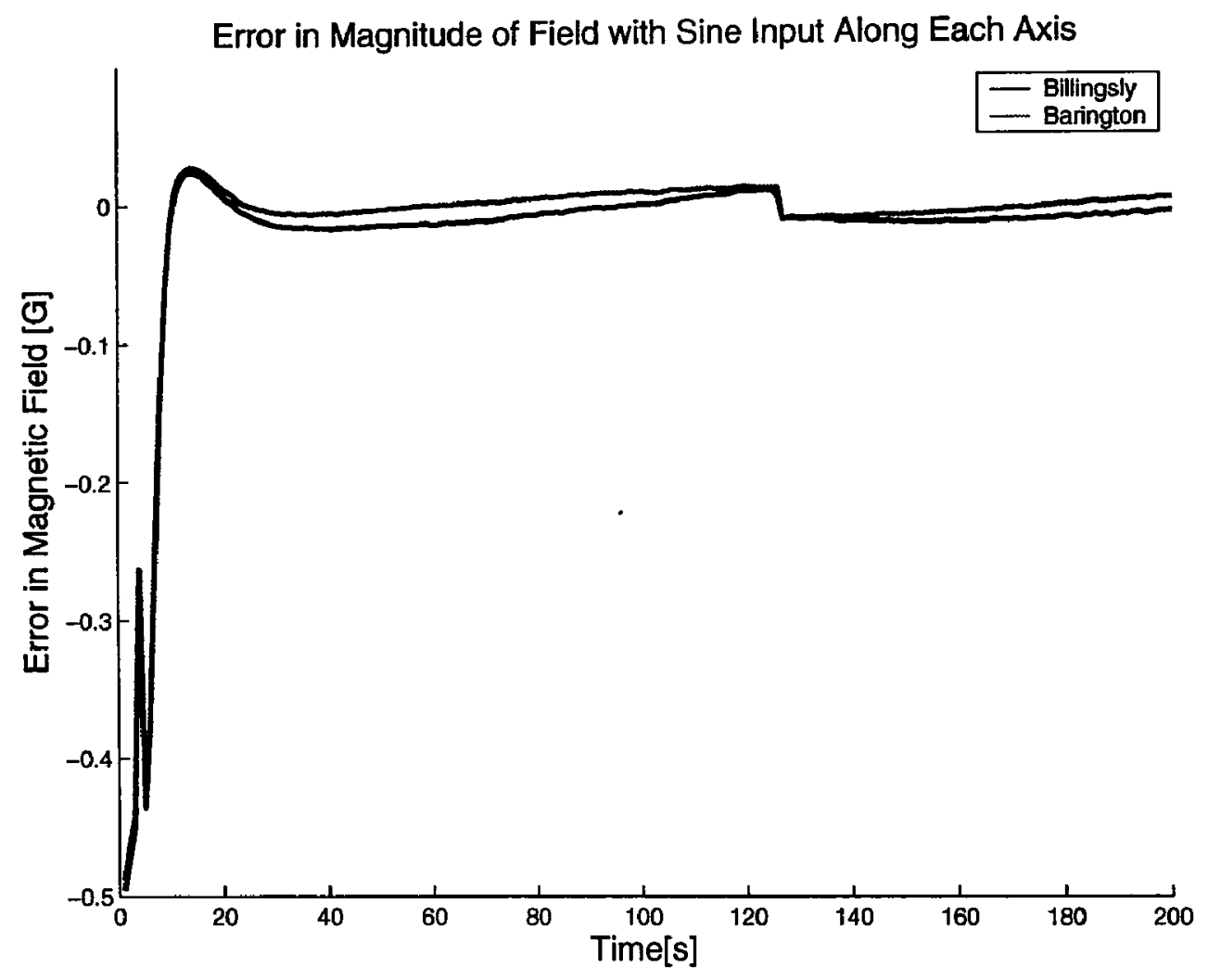

Figure 20: Error in Magnitude of Field with sine Input Alone Each Axis 


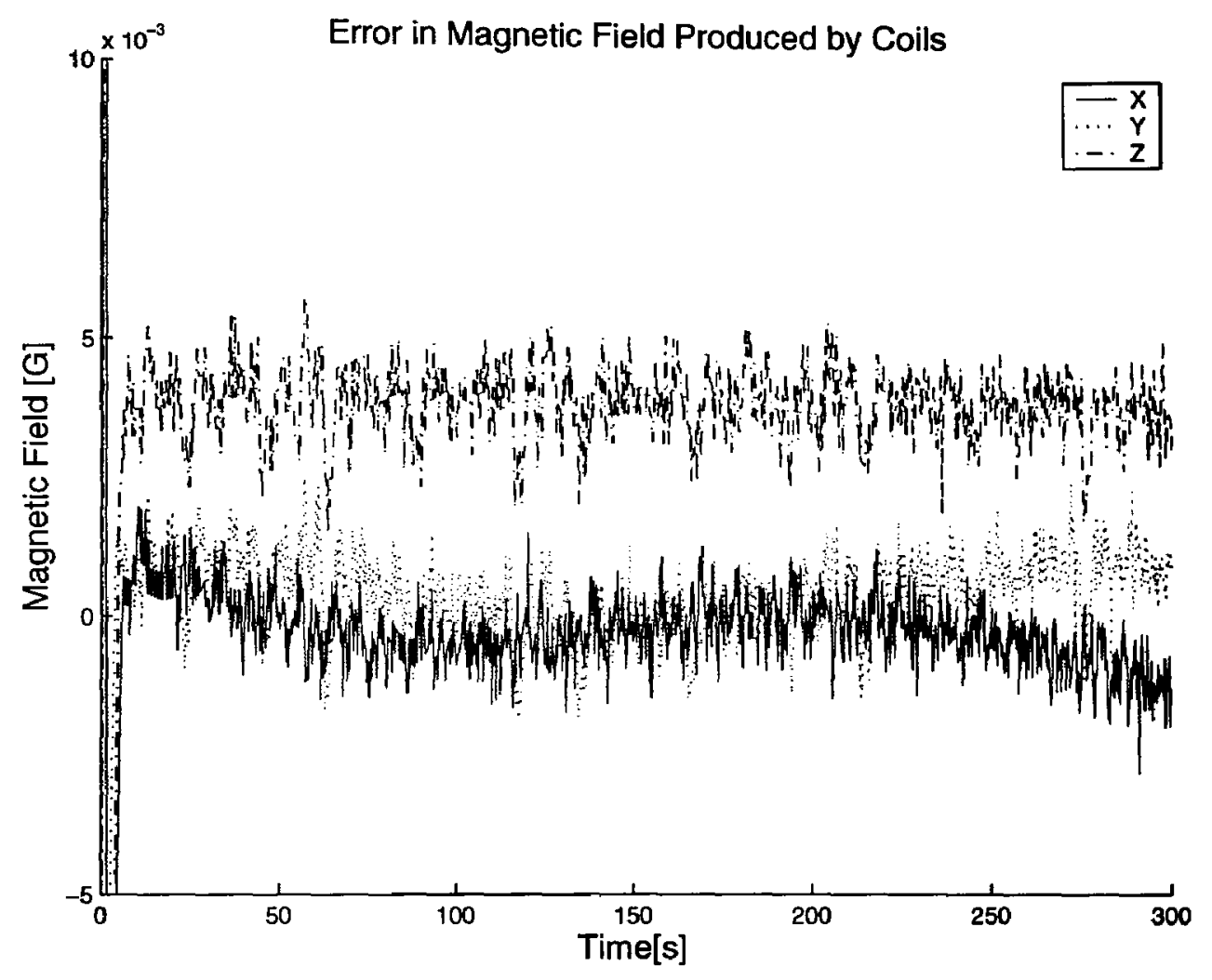

Figure 21: Magnitude of Field 


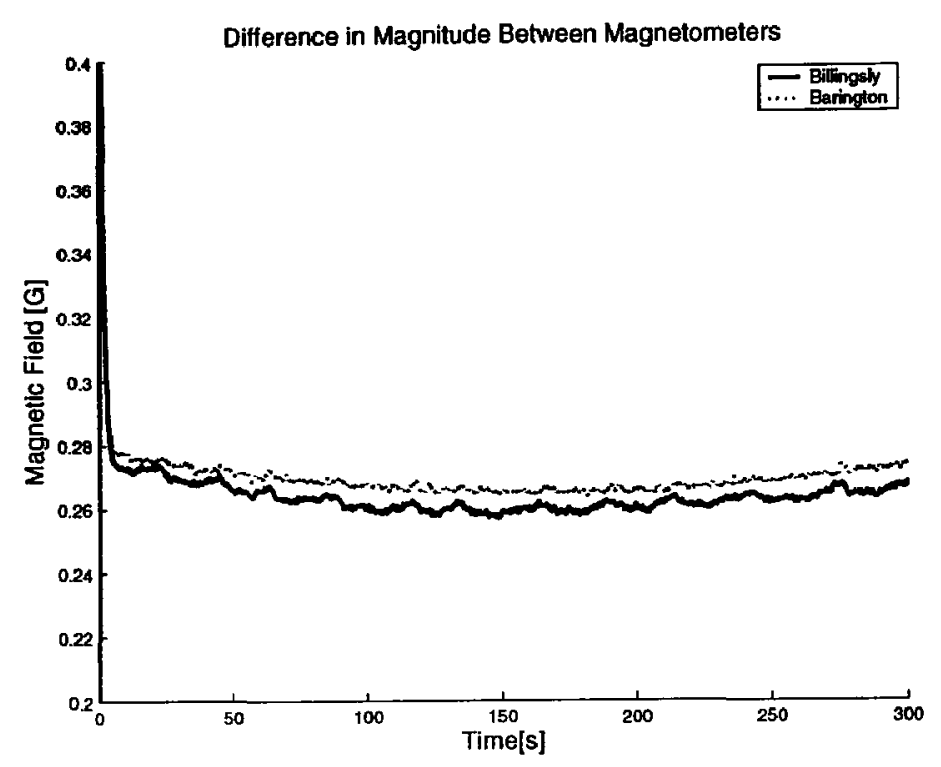

Figure 22: Magnitude of Field

\subsubsection{Closed Loop Simulation}

After it was determined that the Billingsly magnetometer was capable of following the magnetic field laid out by the flight simulation, the loop was closed and the magnetometer readings were fed into the control algorithm of the simulation.

Performance was indistinguishable from the previous trial (Figure 23, leading to the conclusion that the control algorithm and incorporated filter were capable of dealing with the noise that was being produced.

\subsection{B-dot Control Law}

It was already determined that a B-dot controller would be efficient to bring the satellite to a controllable state, so several different gains were tested on a trial and error basis. Refer to Table 8 for the list of gains, and their respective times to slow the satellite to $1 \% \mathrm{~s}$ on all axes (hence reaching Mode 20 of the QuickSat simulation). 


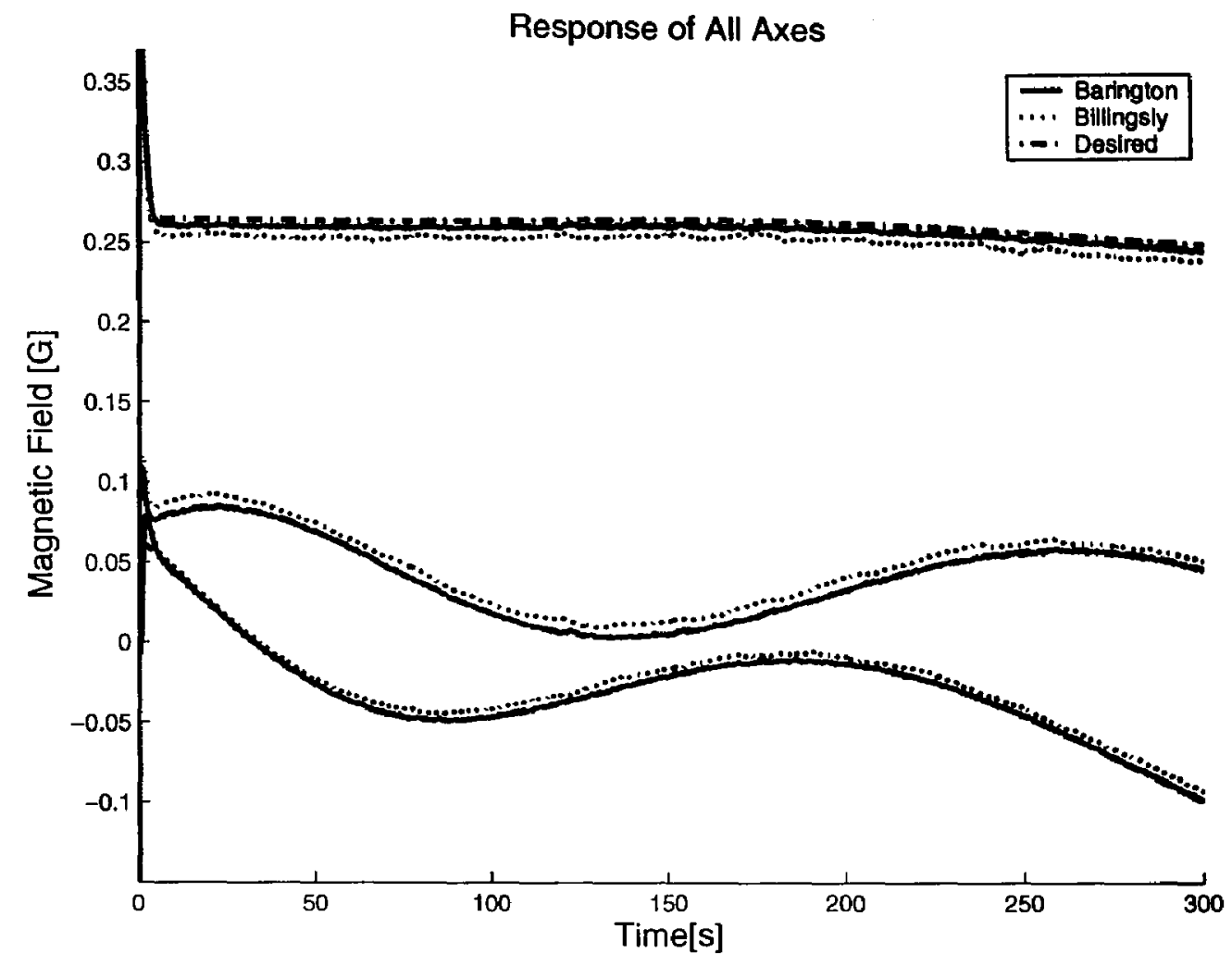

Figure 23: Response of Each Axis to Simulation Input With Billingsly Magnetometer Data Driving Simulation

\begin{tabular}{|c|c|c|}
\hline \multirow{2}{*}{ K,Gain Coefficient } & \multicolumn{2}{|c|}{ Time to Reach Mode 20 [s] } \\
\cline { 2 - 3 } & Trial 1 & Trial 2 \\
\hline$\frac{1}{|B|}$ & 598 & 517 \\
$\frac{1}{|B|^{2}}$ & 532 & 460 \\
$\frac{1}{|B|^{3}}$ & 900 & 520 \\
$\frac{1}{|B|^{4}}$ & 1018 & - \\
$\frac{1}{|\hat{b}|}$ & 520 & - \\
\hline
\end{tabular}

Table 8: B-Dot Controller Coefficients 
A fixed set of arbitrarily chosen initial conditions was used to determine which control law slowed the satellite and moved it from the de-tumbling phase. The initial roll, pitch and yaw velocities were $1,0.5,0.5 \% \mathrm{~s}$ respectively.

While all the coefficients were able to stabilize the satellite, the one that achieved this task consistently with the minimum time required was chosen, and the resulting control became

$$
\mathbf{m}=\frac{\dot{\mathrm{B}}}{|\mathbf{B}|^{2}}
$$

\subsubsection{Stability of B-dot Control Law}

To ensure that the B-dot control law was capable of handling various initial conditions, the simulation was repeated with the above control law for a variety of initial tumbling rates.

In Table 9, it should be noted that even in the case of tumbling rates as high as $10 \%$ on each axis, resulting in an overall rate of $31.63 \%$, the B-dot control law was able to stabilize the spacecraft and enter into Mode 20 within a reasonable time frame of 97 minutes, which is equivalent to a little more than one orbit.

\begin{tabular}{|c|c|c|c|}
\hline Roll $[\% / s]$ & Pitch $[\% / s]$ & Yaw $[\% / s]$ & Time $[\mathrm{s}]$ \\
\hline 0.1 & 0.1 & 1.0 & 292 \\
1.0 & 1.0 & 1.0 & 376 \\
2.0 & 2.0 & 2.0 & 850 \\
3.0 & 3.0 & 3.0 & 952 \\
5.0 & 5.0 & 5.0 & 2800 \\
10.0 & 10.0 & 10.0 & 5830 \\
\hline
\end{tabular}

Table 9: Various Initial Tumbling Rates and their Corresponding Time to Reach Mode 20

Finally, simulations were completed to demonstrate the magnetometers ability throughout all modes of the spacecrafts lifetime (Figure 24). 


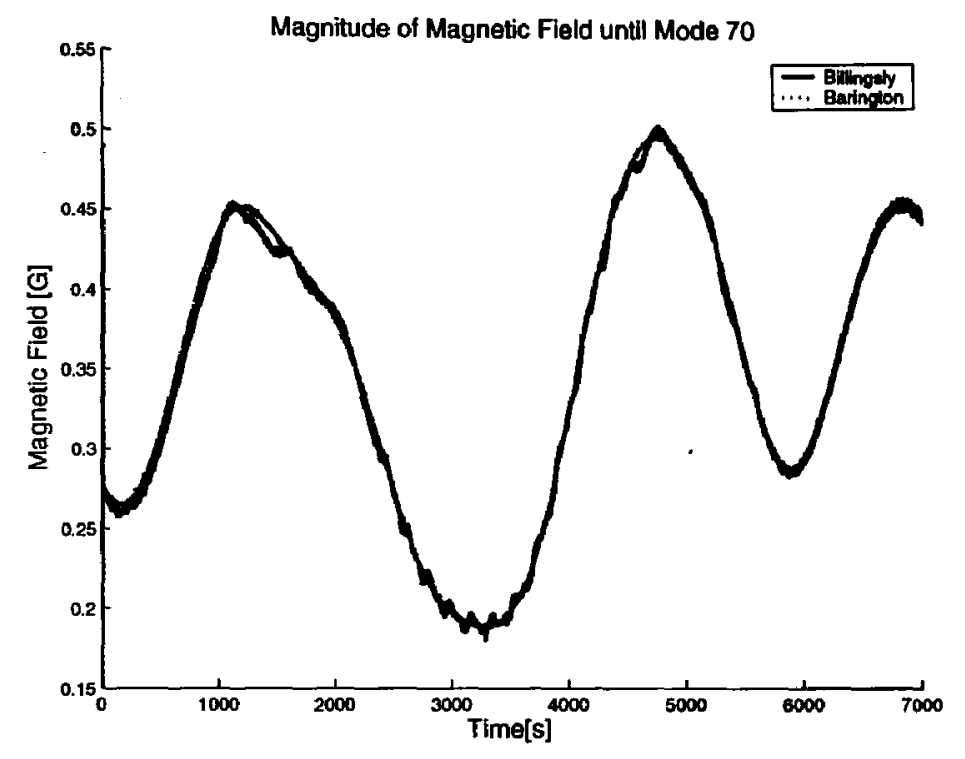

Figure 24: Magnitude of the Magnetic Field with Simulation Running until Mode 70 


\section{Controller Design for RACE project}

RACE was controlled using a standard PID controller and the states were estimated using a Kalman filter.

\subsection{PID Controller}

A proportional plus derivative controller was implemented to control the RACE platform. The feedback in terms of error in position, $e=\theta-\theta_{\text {desired }}$, follows the form

$$
u=K_{p} e+K_{d} \frac{d e}{d t}+K_{i} \int e d t
$$

The proportional, $K_{p}$, derivative $K_{d}$, and integral $K_{i}$ gains were initially determined in simulation and fine tuned during hardware simulations.

\subsection{Kalman Filter}

A Kalman filter is a common tool used on many spacecraft to estimate position and velocity when multiple and redundant sensors of varying degrees of accuracy and availability are being used for attitude and orbit determination. The following is a brief summary of the development of a standard Kalman filter with time invariant system matrices. A Kalman filter utilizes a linear combination of state estimation, $\hat{\mathbf{x}}_{k}$ and the weighted difference between the actual measurement, $\mathbf{z}$, and the measurement prediction, $\mathbf{H} \mathbf{x}$.

$$
\hat{\mathbf{x}}_{k}^{+}=\hat{\mathbf{x}}_{k}^{-}+\mathbf{K}\left(\mathbf{z}-\mathbf{H} \hat{\mathbf{x}}_{k}^{-}\right)
$$

The notation, $\hat{\mathbf{x}}_{k}^{+}$, signifies the state estimation directly after the measurement is taken and, $\hat{\mathbf{x}}_{k}^{-}$indicates the estimation directly before. The residual is defined as $\left(\mathbf{z}-\mathbf{H} \hat{\mathbf{x}}_{k}^{-}\right)$, and it is desirable to have this value as close to zero as possible. 
For a discreet system, the state at each time step, $\mathbf{x}_{k+1}$, can be defined in terms of the value at the previous time step, $\mathbf{x}_{k}$, as

$$
\mathbf{x}_{k+1}=\phi_{k} \mathbf{x}_{k}+\mathbf{w}_{k}
$$

where, $\mathbf{w}_{k}$ is the process noise vector, and $\phi_{k}$ the state transition matrix that is defined later in this section. The process noise vector is assumed as white noise with the following mean and covariance,

$$
\begin{aligned}
& E\left[\mathbf{w}_{k}\right]=0 \\
& E\left[\mathbf{w}_{k} \mathbf{w}_{i}^{T}\right]=\mathbf{Q}_{k} \delta_{k k}
\end{aligned}
$$

The measurement vector can be related to the state vector as

$$
\mathbf{z}_{k}=\mathbf{H}_{k} \mathbf{x}_{k}+\nu_{k}
$$

where, $\nu_{k}$ is the measurement noise vector and has mean and covariance values of

$$
\begin{aligned}
& E\left[\boldsymbol{\nu}_{k}\right]=0 \\
& E\left[\boldsymbol{\nu}_{k} \boldsymbol{\nu}_{i}^{T}\right]=\mathbf{R}_{k} \delta_{k k}
\end{aligned}
$$

The covariance of the state estimate directly after the measurement, $\mathbf{P}_{k}^{+}$, is defined in terms of error, e as

$$
\mathbf{P}_{k}^{+}=E\left[\mathbf{e}_{k}^{+} \mathbf{e}_{k}^{+T}\right]
$$

Also it follows that the covariance directly before measurement, $\mathbf{P}_{k}^{-}$, is

$$
\mathbf{P}_{k}^{-}=E\left[\mathbf{e}_{k}^{-} \mathbf{e}_{k}^{-T}\right]
$$


where the error term is defined as

$$
\mathbf{e}_{k}^{+} \equiv \mathbf{x}_{k}-\hat{\mathbf{x}}_{k}^{+}
$$

The error term can then be written as

$$
\begin{aligned}
& \mathbf{e}_{k}^{+}=\mathbf{x}_{k}-\hat{\mathbf{x}}_{k}^{-}-\mathbf{K}_{\mathbf{k}} \mathbf{z}_{k}+\mathbf{K}_{k} \mathbf{H}_{k} \hat{\mathbf{x}}_{k}^{-} \\
& \mathbf{e}_{k}^{+}=\left(\mathbf{I}-\mathbf{K}_{k} \mathbf{H}_{k}\right)\left(\mathbf{x}_{k}-\hat{\mathbf{x}}_{k}^{-}\right)-\mathbf{K}_{k} \boldsymbol{\nu}_{k} \\
& \mathbf{e}_{k}^{+}=\left(\mathbf{I}-\mathbf{K}_{k} \mathbf{H}_{k}\right) \mathbf{e}_{k}^{-}-\mathbf{K}_{k} \boldsymbol{\nu}_{k}
\end{aligned}
$$

So expanding the covariance matrix results in the following,

$$
\begin{aligned}
& \mathbf{P}_{k}^{+}=E\left[\left(\mathbf{I}-\mathbf{K}_{k} \mathbf{H}_{k}\right) \mathbf{e}_{k}^{-} \mathbf{e}_{k}^{-T}\left(\mathbf{I}-\mathbf{K}_{k} \mathbf{H}_{k}\right)^{T}\right] \\
& -E\left[\left(\mathbf{I}-\mathbf{K}_{k} \mathbf{H}_{k}\right)\left(\mathbf{e}_{k}^{-} \boldsymbol{\nu}_{k}^{T}\right) \mathbf{K}_{k}^{T}\right] \\
& -E\left[\mathbf{K}_{k}\left(\boldsymbol{\nu}_{k} \mathbf{e}_{k}^{-T}\right)\left(\mathbf{I}-\mathbf{K}_{k} \mathbf{H}_{k}\right)^{T}\right] \\
& +E\left[\mathbf{K}_{k} \boldsymbol{\nu}_{k} \boldsymbol{\nu}_{k}^{T} \mathbf{K}_{k}^{T}\right]
\end{aligned}
$$

To simplify the above equation, the definition of the covariances of $\nu_{k}$ and $\mathbf{P}_{k}^{-}$must be applied. As well, the two middle terms go to zero, as white noise is independent of state values, in other words $E\left(\nu_{k} \mathbf{e}_{k}^{-T}\right)=0$. So when simplified, the covariance matrix directly after measurement is

$$
\mathbf{P}_{k}^{+}=\left(\mathbf{I}-\mathbf{K}_{k} \mathbf{H}_{k}\right) \mathbf{P}_{k}^{-}\left(\mathbf{I}-\mathbf{K}_{k} \mathbf{H}_{k}\right)^{T}+\mathbf{K}_{k} \mathbf{R}_{k} \mathbf{K}_{k}^{T}
$$

The Kalman filter gain, $\mathbf{K}_{k}$, must be chosen to minimize the errors in the system. The main terms of interest in the covariance matrix are lined along the diagonal, and hence, the optimal Kalman filter gains are chosen to minimize the trace of $\mathbf{P}$.

Noting first the identity 


$$
\frac{d \operatorname{Tr}[\mathrm{AB}]}{d \mathbf{A}}=\mathbf{B}^{T} \text { and } \frac{d \operatorname{Tr}[\mathbf{A C A}]}{d \mathbf{A}}=2 \mathbf{A C}
$$

The trace of $\mathrm{P}$ can be written as

$$
\begin{aligned}
\frac{d T r\left[\mathbf{P}_{k}^{+}\right]}{d \mathbf{K}_{k}} & =\frac{d}{d \mathbf{K}_{k}}\left[\operatorname{Tr}\left(\left(\mathbf{I}-\mathbf{K}_{k} \mathbf{H}_{k}\right) \mathbf{P}_{k}^{-}\left(\mathbf{I}-\mathbf{K}_{k} \mathbf{H}_{k}\right)^{T}+\mathbf{K}_{k} \mathbf{R}_{k} \mathbf{K}_{k}^{T}\right)\right] \\
\frac{d T r\left[\mathbf{P}_{k}^{+}\right]}{d \mathbf{K}_{k}} & =\frac{d}{d \mathbf{K}_{k}}\left[\operatorname{Tr}\left(\mathbf{P}_{k}^{-}-\mathbf{K}_{k} \mathbf{H}_{k} \mathbf{P}_{k}^{-}-\mathbf{P}_{k}^{-T} \mathbf{H}_{k}^{T} \mathbf{K}_{k}^{T}+\mathbf{K}_{k}\left[\mathbf{H}_{k} \mathbf{P}_{k}^{-T} \mathbf{H}_{k}^{T}+\mathbf{R}_{k}\right]\right)\right] \mathbf{K}_{k}^{T} \\
\frac{d T r\left[\mathbf{P}_{k}^{+}\right]}{d \mathbf{K}_{k}} & =-2\left(\mathbf{H}_{k}\right)^{T}+2 \mathbf{K}_{k}\left(\mathbf{H}_{k} \mathbf{P}_{k}^{-T} \mathbf{H}_{k}^{T}+\mathbf{R}_{k}\right)=0
\end{aligned}
$$

Solving the above for $\mathbf{K}_{k}$, the desired Kalman filter gain is,

$$
\mathbf{K}_{k}=\mathbf{P}_{k}^{-} \mathbf{H}_{k}^{T}\left(\mathbf{H}_{k} \mathbf{P}_{k}^{-T} \mathbf{H}_{k}^{T}+\mathbf{R}_{k}\right)^{-1}
$$

The second part of the Kalman filter consists of propagating the state estimate through the time step. At the beginning of the time step, the state estimate is

$$
\hat{\mathbf{x}}_{k+1}^{-}=\phi \hat{\mathbf{x}}_{k}^{+}
$$

where $\phi$ is the state transition matrix and is defined as

$$
\phi(t)=e^{\mathbf{F} t}=\mathscr{L}^{-1}\left\{[s \mathbf{I}-\mathbf{F}]^{-1}\right\}
$$

The error in this state estimate can be defined by

$$
\begin{aligned}
& \mathbf{e}_{k+1}^{-}=\mathbf{x}_{k+1}-\hat{\mathbf{x}}_{k+1}^{-} \\
& \mathbf{e}_{k+1}^{-}=\phi \mathbf{x}_{k}+\mathbf{w}_{k}-\phi \hat{\mathbf{x}}_{k}^{+} \\
& \mathbf{e}_{k+1}^{-}=\phi\left(\mathbf{x}_{k}-\mathbf{x}_{k}^{+}\right)+\mathbf{w}_{k} \\
& \mathbf{e}_{k+1}^{-}=\phi \mathbf{e}_{k}^{+}+\mathbf{w}_{k}
\end{aligned}
$$


which leads to the following covariance matrix, $\mathbf{P}_{k+1}^{-}$

$$
\mathbf{P}_{k+1}^{-}=E\left[\mathbf{e}_{k+1}^{-} \mathbf{e}_{k+1}^{-T}\right]
$$

Expanding gives

$$
\mathbf{P}_{k+1}^{-}=E\left[\phi \mathbf{e}_{k}^{+} \mathbf{e}_{k}^{+T} \phi^{T}+\phi \mathbf{e}_{k}^{+} \mathbf{w}_{k}^{T}+\mathbf{w}_{k} \mathbf{e}_{k}^{+T} \phi^{T}+\mathbf{w}_{k} \mathbf{w}_{k}^{T}\right]
$$

The expectation of $\mathbf{w}_{k} \mathbf{e}_{k}^{+T}$ is zero and the expectation of $\mathbf{w}_{k} \mathbf{w}_{k}^{T}$ is equivalent to the matrix, $\mathrm{Q}_{k}$, therefore the recursive covariance matrix can be calculated as follows.

$$
\mathbf{P}_{k+1}^{-}=\phi \mathbf{P}_{k}^{+} \phi+\mathbf{Q}_{k}
$$

These are the standard equations needed for a Kalman filter, and can be summarized in Figure 25, however as noted previously, the error states are of interest in this study and hence these standard equations have been slightly altered to use the error states.

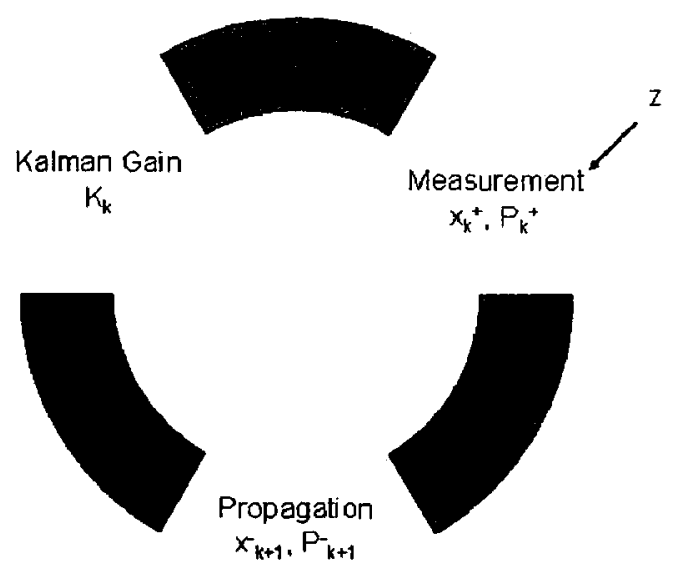

Figure 25: Summary of Kalman Filter 
The error vector after measurement based on the above definition is

$$
\begin{aligned}
& \mathbf{e}_{k}^{+}=\mathbf{x}_{k}-\hat{\mathbf{x}}_{k}^{+} \\
& \mathbf{e}_{k}^{+}=\mathbf{x}_{k}-\hat{\mathbf{x}}_{k}^{-}+\mathbf{K}_{k}\left(\mathbf{z}_{k}-\mathbf{H}_{k} \hat{\mathbf{x}}_{k}^{-}\right) \\
& \mathbf{e}_{k}^{+}=\mathbf{e}_{k}^{-}+\mathbf{K}_{k}\left(\mathbf{z}_{k}-\mathbf{H}_{k}\left[\mathbf{x}_{k}-\mathbf{e}_{k}^{-}\right]\right) \\
& \mathbf{e}_{k}^{+}=\mathbf{e}_{k}^{-}+\mathbf{K}_{k}\left(\mathbf{z}_{k}-\mathbf{H}_{k} \mathbf{x}_{k}+\mathbf{H}_{k} \mathbf{e}_{k}^{-}\right)
\end{aligned}
$$

Defining a new measurement variable, $\tilde{\mathbf{z}}=\mathbf{z}_{k}-\mathbf{H}_{k} \mathbf{x}_{k}$, as the error measurement, the error state estimate becomes

$$
\hat{\mathbf{e}}_{k}^{+}=\hat{\mathbf{e}}_{k}^{-}+\mathbf{K}_{k}\left(\tilde{\mathbf{z}}_{k}-\mathbf{H}_{k} \hat{\mathbf{e}}_{k}^{-}\right)
$$

Before the measurement, the error was previously defined as

$$
\mathbf{e}_{k+1}^{-}=\phi \mathbf{e}_{k}^{+}+\mathbf{w}_{k}
$$

Therefore, the estimate of the error state is

$$
\hat{\mathbf{e}}_{k+1}^{-}=\phi \mathbf{e}_{k}^{+}
$$

The covariance matrices as well as the gain matrix, remain the same as previously stated.

\section{RACE Simulations}

Before testing of the Kalman filter could begin, it was necessary to determine several of the characteristics of the system and determine confidently that the sensors used in RACE were functioning properly and were capable of the tests that were to be carried out. Once the system was characterized, simulations of the controller and Kalman filter were run to determine the optimal coefficients needed for the system. The controller was then implemented 
in the RACE software and hardware tests were carried out. Finally the Kalman filter was added to the system and was tested.

\subsection{Rate Sensor Bias}

As the rate sensor had a strong dependence on the temperature, several tests were carried out to determine the effects of the room temperature and ambient temperature drift on the sensor. Firstly, the system was powered up but held in place while the rate sensor took measurements, this test was carried out several times over for a variety of testing times, the longest occurring for 1 hour. Find below in Figure 26 the results of this testing that occurred over a 20 minute period.

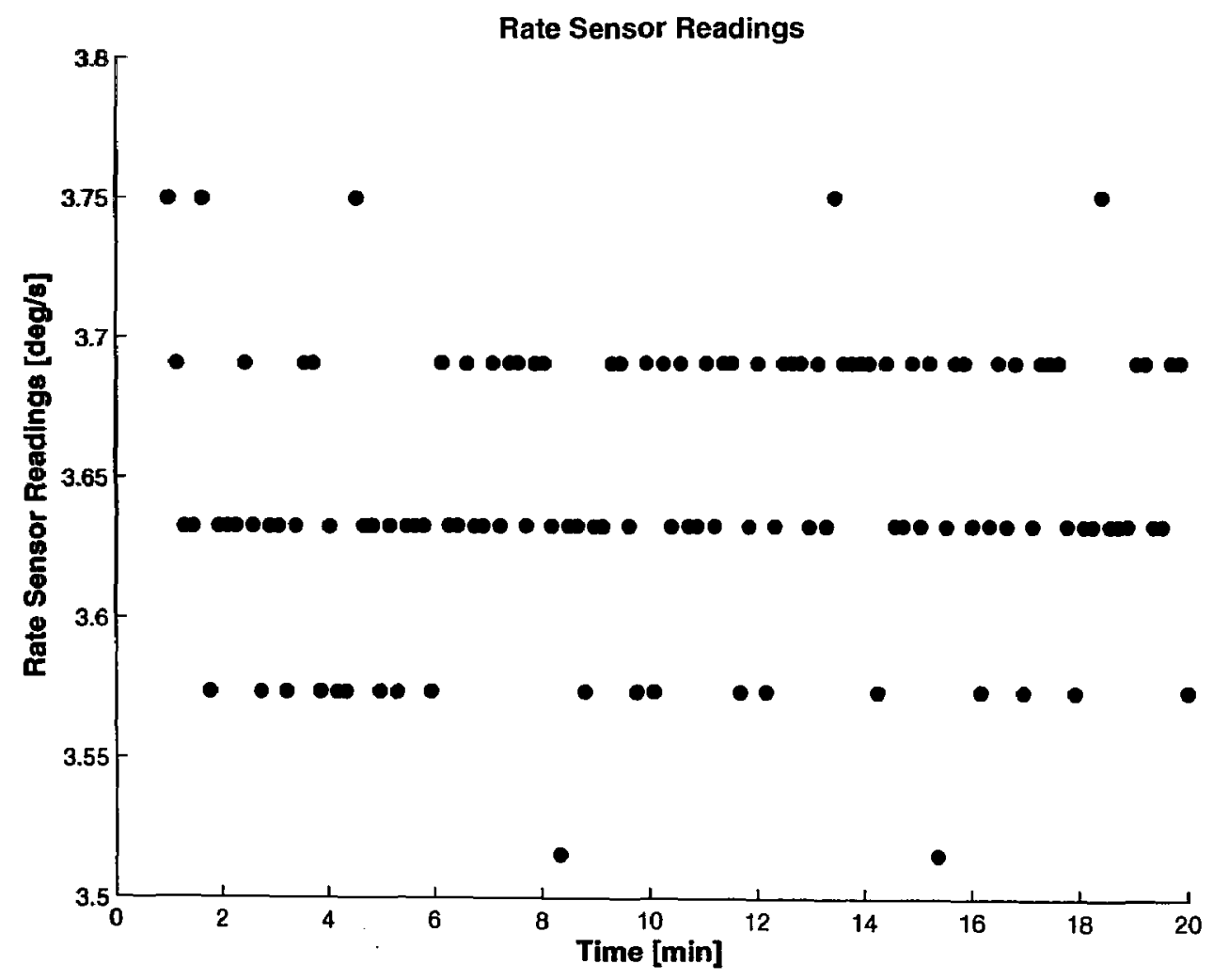

Figure 26: Rate Sensor Readings while RACE is fixed and actuators are powered off 
The natural uncalibrated bias of the rate sensor, as well as the discreet values through which the rate sensor fluctuates can be seen. It appeared that there was no room temperature drift within the test facility, or whatever small fluctuations in temperature that occurred were negligible with regard to the sensitivity of the rate sensor.

Ambient temperature drift was also determined to be a factor that might inhibit proper functioning of the rate sensor. Again, the platform was held in place, and the momentum wheel and the air thruster were turned on to create heat within the system, refer to Figure 27.

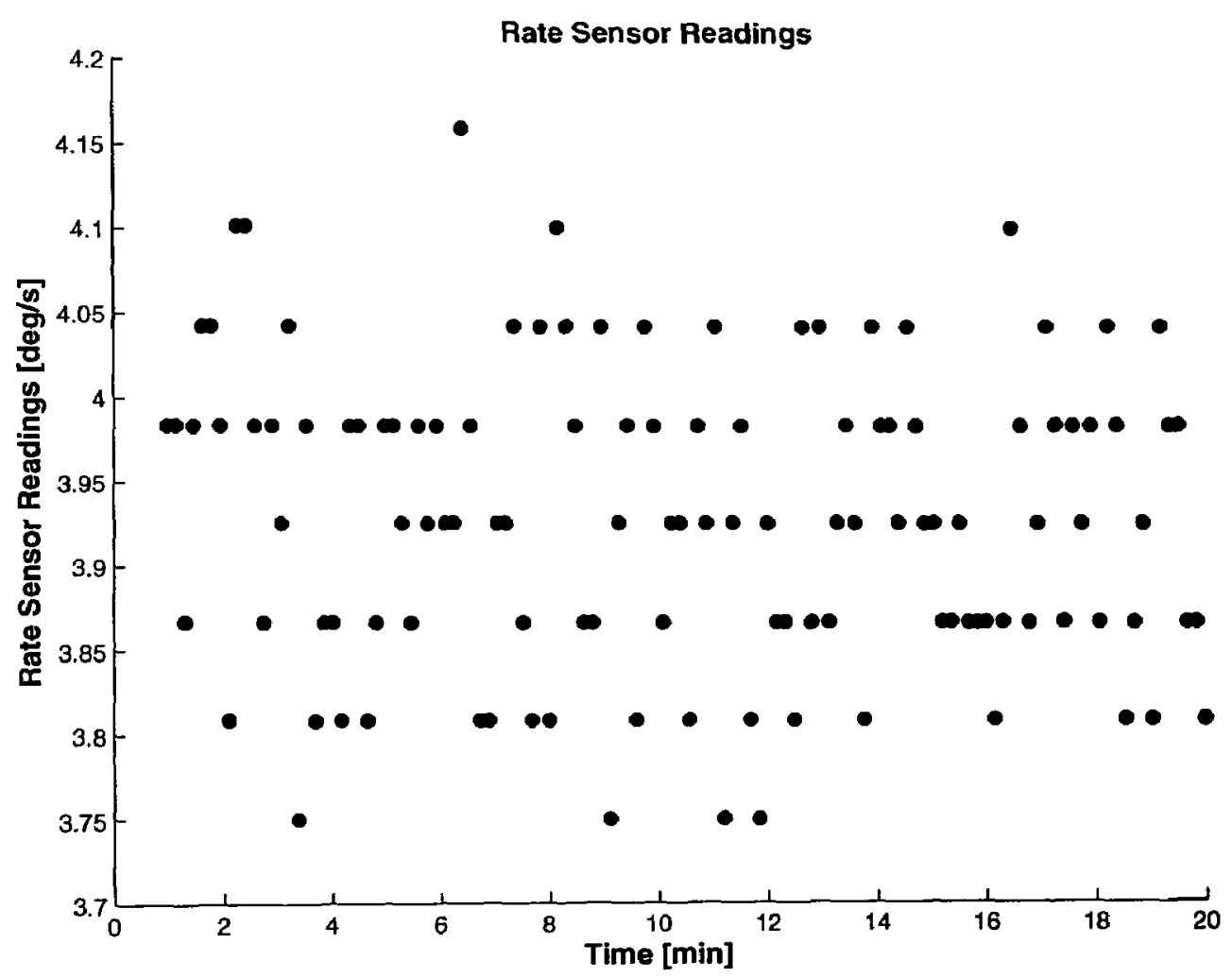

Figure 27: Rate Sensor Readings while RACE is fixed and actuators are powered.

The heat created by the momentum wheel and air thruster did not affect the rate sensor measurements.

As no effect was noticed in either of the previous tests, a qualitative test was done on the 
rate sensor to determine if in fact the temperature dependance as stated by the manufacture existed. Thus, a heater was used to heat the ambient air around RACE and while held static once more, a series of rate sensor measurements were taken.

Figure 28 demonstrates the inverse relationship that indeed exists between the temperature and rate sensor measurements. Although the platform was fixed, the rate sensor readings showed a decreasing angular rate until a saturation point of approximately $-0.5 \% \mathrm{~s}$ when the ambient temperature was increased.

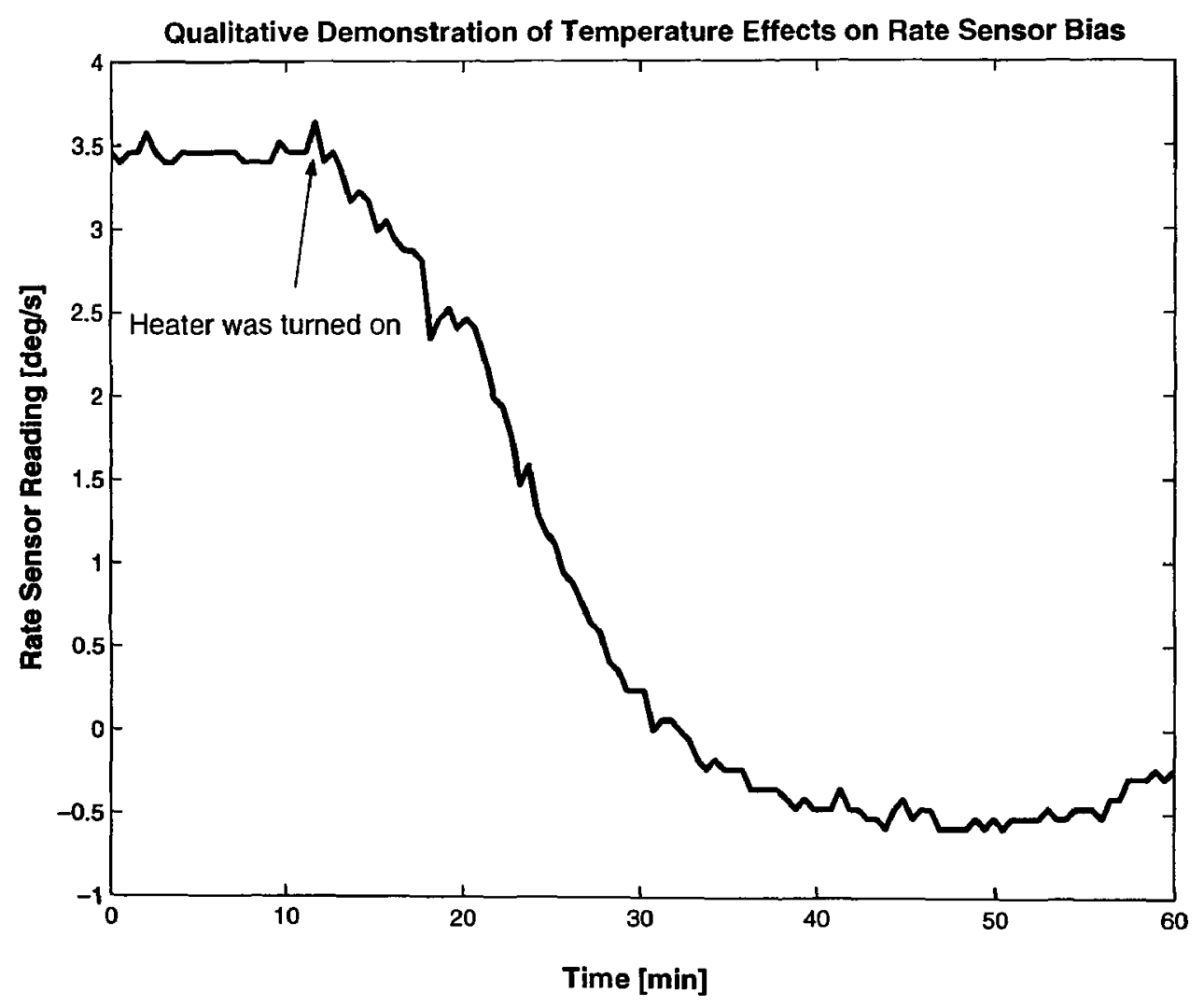

Figure 28: Effects of Increased Temperature on Rate Sensor

Two points can be made from this result, the first is that the rate sensor was susceptible to ambient temperature changes. The second is that the heat created by the internal RACE computer and other components would not compromise the accuracy of the rate sensor 
measurements.

Finally the output from the rate sensor was compared to the output from the base encoder, Figure 29. The momentum wheel was powered up to a rate of $3000 \mathrm{rpm}$ and the RACE platform was left to spin freely. The measurement frequency for both sensors was $1 \mathrm{~Hz}$, and the tests were carried out for 5 minutes.

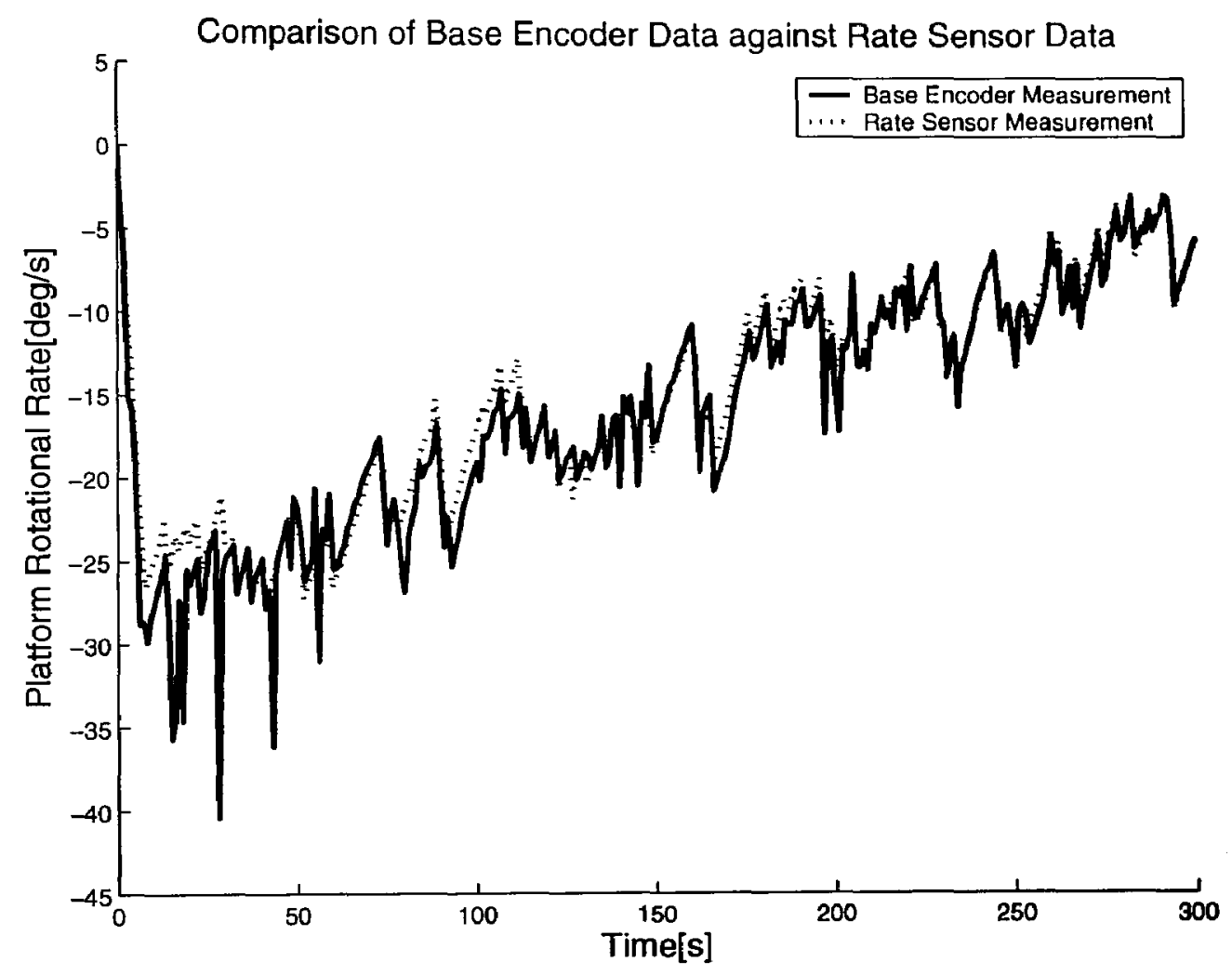

Figure 29: Angular Rates of a free spinning RACE platform

\subsection{Controller Testing}

The RACE platform PID controller was designed in simulation to minimize both percent overshoot and steady state error. Once parameters were chosen in simulation, they were fine 


\begin{tabular}{|c|c|c|c|c|}
\hline$K_{p}$ & $K_{i}$ & $K_{d}$ & Percent Overshoot & $e_{s s}$ \\
\hline 1.2 & 0 & 0.5 & 76 & 0.75 \\
\hline 1.2 & 0 & 0.75 & 40 & 0.93 \\
\hline 1.4 & 0 & 0.75 & 52 & 1.40 \\
\hline 1.6 & 0 & 0.75 & 56 & 0.36 \\
\hline 1.2 & 0 & 0.8 & 53 & 1.05 \\
\hline 1.2 & 0.1 & 0.8 & 50 & 0.05 \\
\hline 1.2 & 0.1 & 0.6 & 85 & 1.40 \\
\hline 1.2 & 0.2 & 0.8 & 80 & 0.05 \\
\hline
\end{tabular}

Table 10: Robust Controller Gains for Helmholtz Cage

tuned using hardware testing. Refer to Table 10 for a variety of the gains tested and the corresponding percent overshoot and steady state error $\left(e_{s s}\right)$ that occurred. All tests were carried out for 100 seconds at a measurement frequency of $2 \mathrm{~Hz}$ with a step input of $15^{\circ}$.

The final controller chosen had gains $K_{p}=1.2, K_{i}=0.1$ and $K_{d}=0.8$. There was a $50 \%$ overshoot in the response with a steady state error of approximately $0.05^{\circ}$. The time to settle to its final value was approximately 15 seconds. Refer to Figure 30.

\subsection{Kalman Filter Testing}

The components of the Kalman filter that were adapted to the RACE system were the measurement noise matrix, $\mathbf{R}$ and the process noise matrix, $\mathbf{Q}$. The measurement noise matrix was a diagonal $2 \times 2$ matrix, as the noise levels in the two sensors on board RACE were uncorrelated. The noise level in the base encoder was chosen as the precision of the angular position measurement, determined by the size of each step, $0.0031 \mathrm{rad}$. The noise level in the output of the rate sensor was a manufacturer specified quantity.

$$
\mathbf{R}=\left[\begin{array}{cc}
0.0031 & 0 \\
0 & 4.36 e-5
\end{array}\right]
$$


The process noise matrix was a $3 \times 3$ matrix that contained only one non-zero value that was the manufacturer stated rate sensor bias.

$$
\mathbf{Q}=\left[\begin{array}{ccc}
0 & 0 & 0 \\
0 & 0 & 0 \\
0 & 0 & 1.75 e-6
\end{array}\right]
$$

The initial estimates for the Kalman filter were chosen arbitrarily as

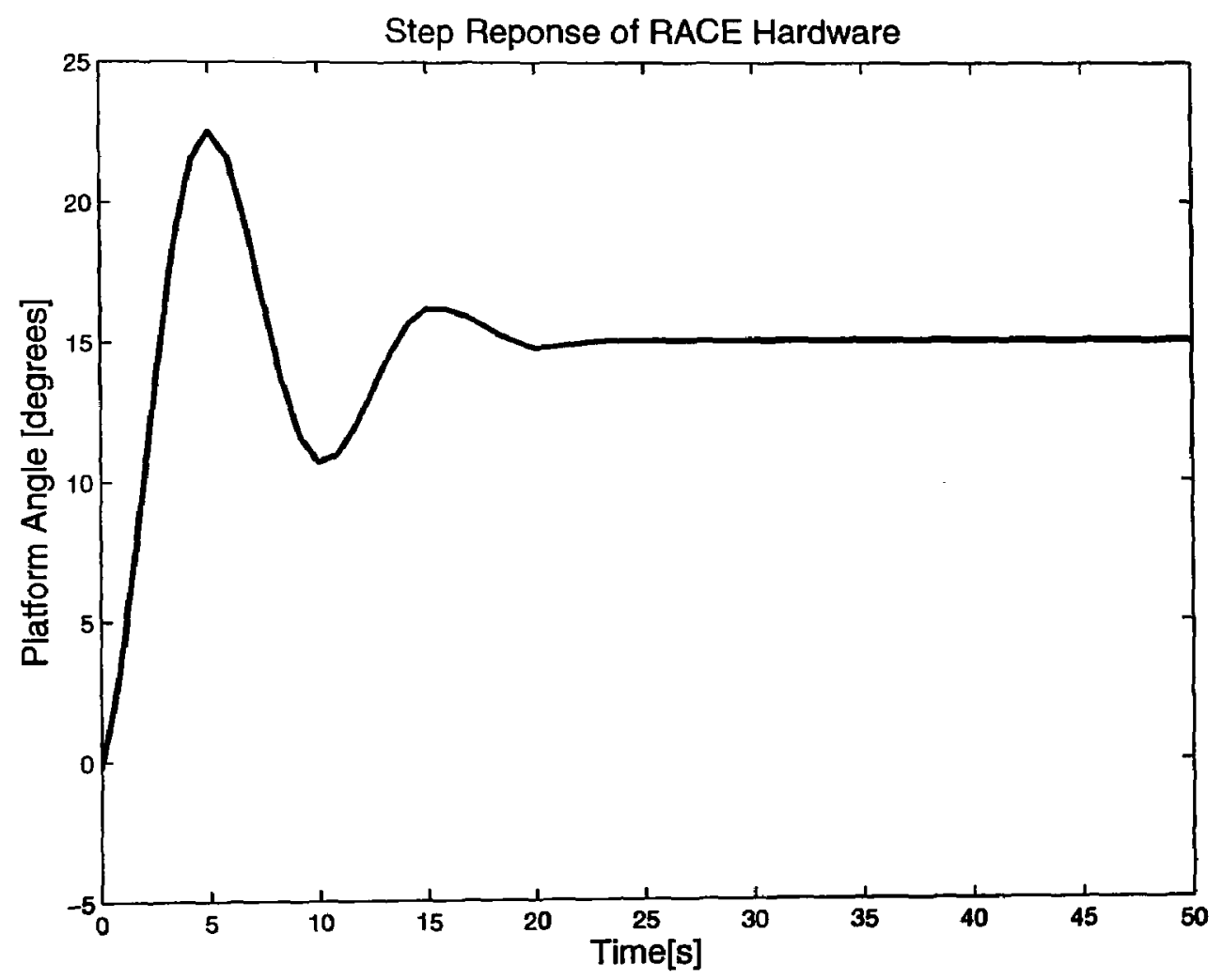

Figure 30: Response of RACE to a Step Input 


$$
\begin{gathered}
\mathbf{e}_{0}=\left[\begin{array}{c}
1.0 \\
0.1 \\
0.01
\end{array}\right] \\
\mathbf{P}_{0}=\left[\begin{array}{lll}
1.0 & 0.1 & 0.2 \\
0.1 & 1.0 & 0.2 \\
0.1 & 0.2 & 1.0
\end{array}\right]
\end{gathered}
$$

The RACE hardware tests were carried out for 225 second intervals, the sampling frequency of the rate sensor was constant at $1 \mathrm{~Hz}$, while the sampling frequency of the base encoder was gradually decreased to assess the Kalman filter's ability to deal with missing data. During testing, the momentum wheel was spinning at approximately $3000 \mathrm{rpm}$ and the platform was free to rotate. No controller was implemented during these tests to maintain the desired fixed state at zero degrees; therefore, the error vector that was used to drive the Kalman filter was simply the state vector. Refer to Figure 31 for the results in position and Figure 32 for the results in angular velocity.

The estimate of the position and velocity calculated using the Kalman filter converged to the values determined from the onboard sensors. However, the estimate does not track the actual angular velocity exactly, therefore fine tuning of the Kalman filter parameters is needed to correct this anomaly. 


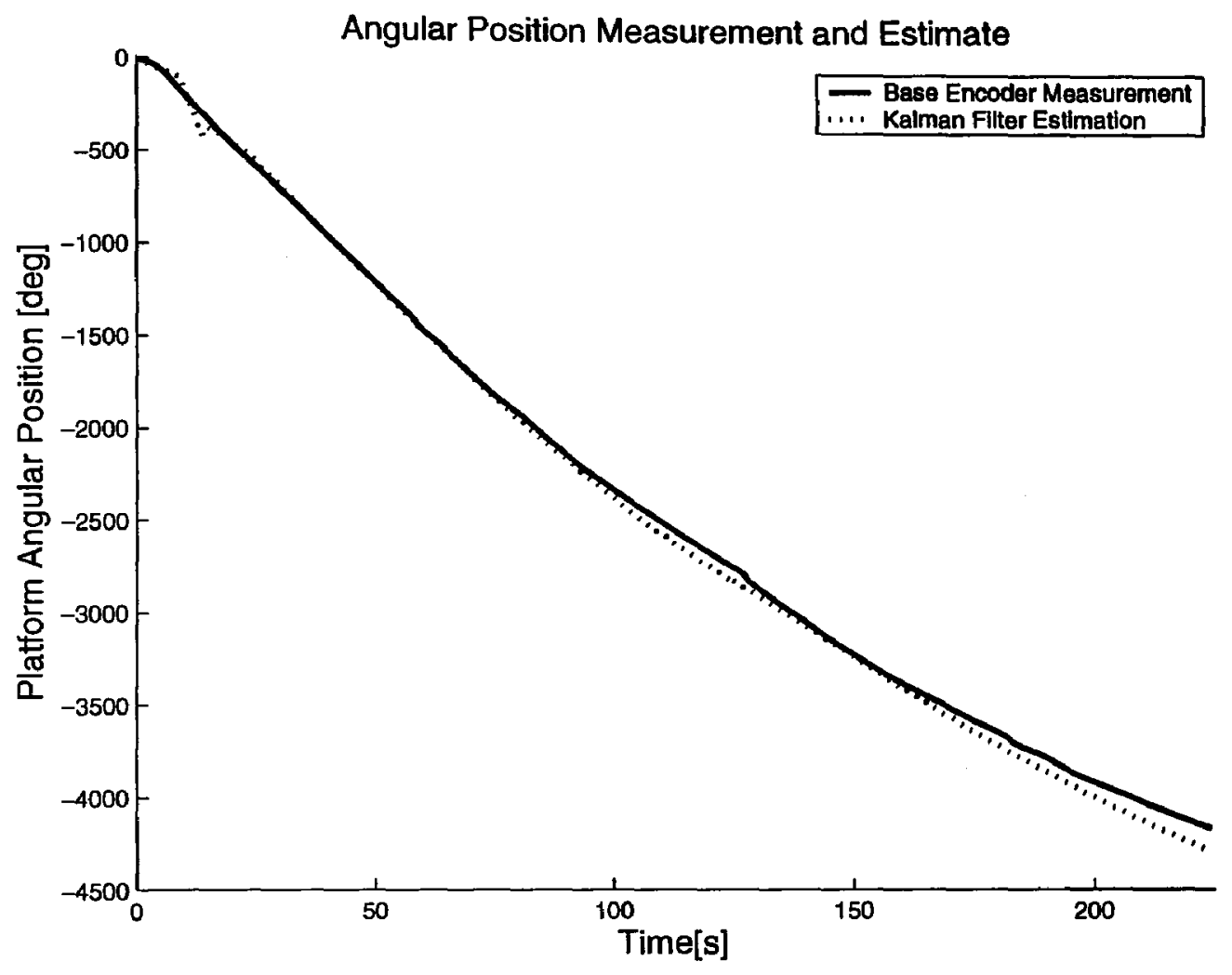

Figure 31: Kalman Filter Estimates of Angular Position (Sampling Frequency of the encoder $=0.2 \mathrm{~Hz}$ ), Angular Position Not Normalized to $2 \pi$ 


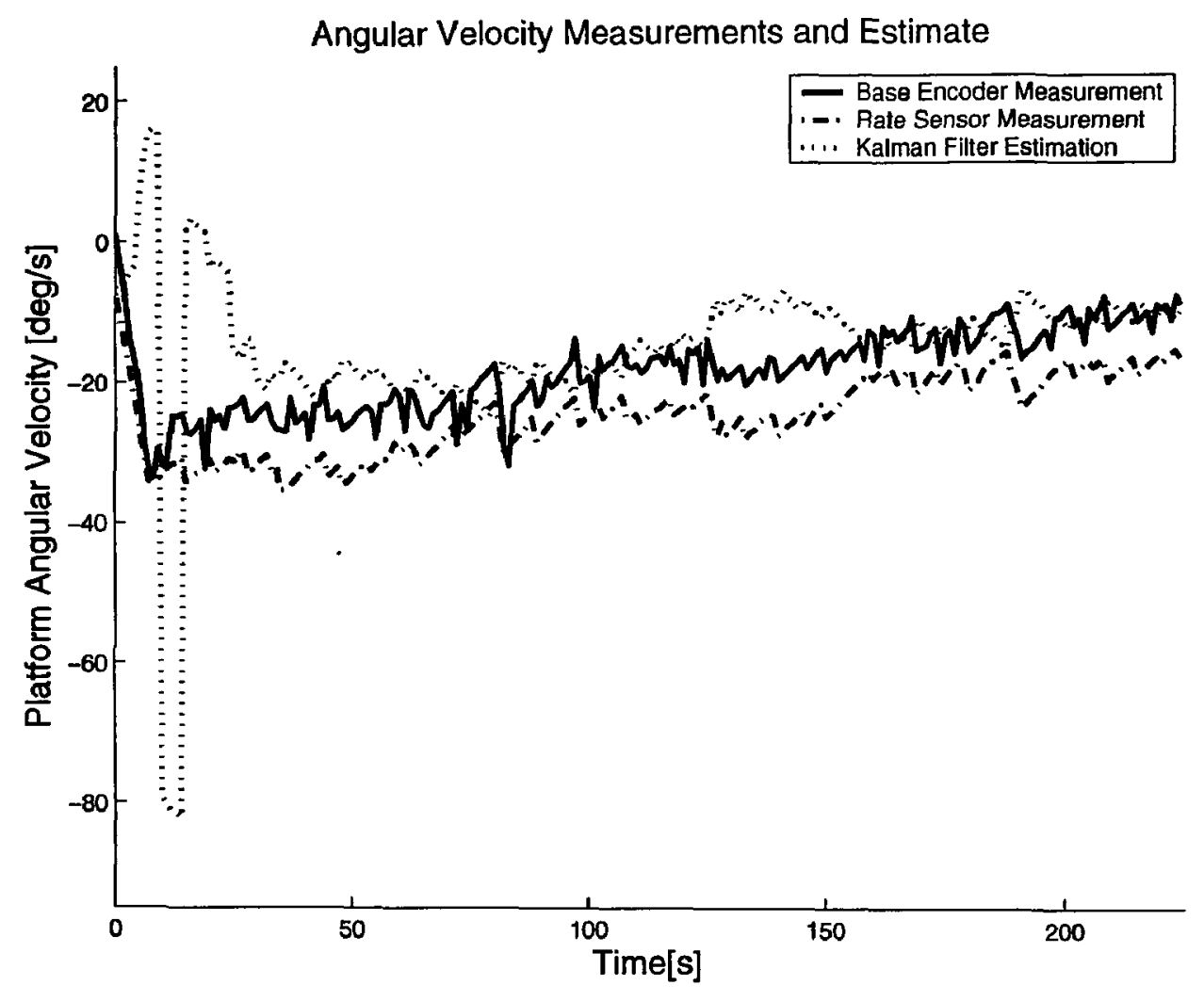

Figure 32: Kalman Filter Estimates of Angular Velocity (Sampling Frequency of the encoder $=0.2 \mathrm{~Hz}$ ) 


\section{Concluding Remarks}

\subsection{QuickSat}

QuickSat, an engineering model designed as a precurser mission for the Canadian Space Agency's small satellite program will be equipped with a magnetometer as a position acquisition instrument. The magnetometer that will be flown aboard QuickSat was tested using three pairs of Helmholtz coils that were erected in 1999 and positioned at $90^{\circ}$ to each other. A satellite simulation, including a magnetic field model, generated the magnetic field values the satellite would experience during orbit, which were in turn created in the test bed.

A support stand was constructed to fix both the magnetometer that was used to control the coils and the one that would be flown onboard QuickSat at the center of the coil configuration. Characterization of the coils was carried out to determine the axis linearity and independence, as well as the transfer function, so that accurate modeling could be completed and proper parameters could be chosen for the robust control law.

Once controllability of the Helmholtz coils was achieved, the flight-rated instrument was added to the test bed. Static measurements - the magnetic field was held constant - were taken to calibrate the magnetometer. Following determination of the proper gain, the magnetometer was then integrated into the hardware in the loop simulation as well, first by reading only, and then fully integrated to drive the attitude control system within the QuickSat simulation. Several different free tumbling rates - considered as the initial conditions of the simulation - were tested. In each case, the magnetometer and control system was capable of stabilizing the spacecraft.

Finally, the control law that was being used to control QuickSat was examined to determine if a more efficient or more powerful controller could be implemented, a b-dot control law was selected. 


\subsection{RACE}

A simulation of the RACE test equipment was completed, and a Kalman filter was implemented on the hardware. Initial hardware testing was carried out, including testing of the onboard sensors, testing of the controller and testing of the Kalman filter while RACE was free to spin.

\subsection{Recommendations for Future Work}

The work that has thus far been completed should be continued to ensure further developments in this field. Improvements on the Helmholtz coil test bed should include the upgrading of the power sources to bi-polar sources, so that the mechanical switch can be removed to simplify the system.

Exhaustive testing should be done to ensure that the magnetometer is indeed flight worthy. Hardware in the loop simulations with a variety of initial conditions should be carried out, ideally reaching the point when the magnetometer fails so that proper and accurate bounds can be put on its abilities.

Regarding RACE, a more complete characterization of the system should be done, including experimental determination of wheel friction coefficients and quantitative testing of the rate sensor temperature bias. Testing of Kalman filter, in conjunction with the controller, needs to be completed to fully validate the Kalman filter. 


\section{References}

[1] (1998) "Billingsley Magnetometers, TFM 100-G2 Miniature Triaxial Fluxgate Magnetometer" Users Manual, Billingsley Magnetics.

[2] (2003) "MR-3 Triaxial Magnetic Field Compensation System, Compensation Coil design and system guide"; Stefan Mayer Instruments.

[3] Davignon, Jean-Philippe (2000) "Status of the Attitude Control System Simulator for Quicksat"; Canadian Space Agency

[4] Brown, R.G. and Hwang, P.Y.C (1997)Introduction to Random Signals and Applied Kalman Filtering, Third Edition; John Wiley and Sons, United States.

[5] Bushenkov, Vladimir et al (2002) "Attitude Stabilization of a Satellite By Magnetic Coils"; Acta Astronautica Vol 50. No 12, pp. 721-728.

[6] Comisel, H. et al (1997) "Attitude Estimation of a Near-Earth Satellite Using Magnetometer Data"; Acta Astronautica Vol 40. No 11, pp. 781-788.

[7] Fortescue, Peter and Stark, John(1995) Spacecraft Systems Engineering Second Edition John Wiley and Sons Ltd.

[8] Franklin, Gene and Powell, David and Emami-Naeini, Abbas (1994)Feedback Control for Dynamic Systems Third Edition; Addison-Wesley Publishing Company Inc, pp. 554-560.

[9] Grassi, M (1997)"Attitude Determination and Control for A Small Remote Sensing Satellite"; Acta Astronautica Vol. 40 No. 9 pp. 675-681.

[10] Grassi, M Vetrella, S Moccia, A (1995) "Preliminary Design of the Attitude Control System of a Microsatellite for Earth Observation";Space Technology Vol 15 No.4 pp. 223-230.

[11] Griffin, Michael; French, James (1991) Space Vehicle Design; American Institute of Aeronautics and Astronautics. 
[12] Herron, Marissa; Goeree, Barry; Shucker, Brian (2000) "Detumbling of UASat" Technical Note University of Arizona.

[13] Janschek, K and Krasilshikov, M (1998) "Minimum Hardware Navigation Concept for LEO Satellites Using Information Fusion"; 12th AIAA Conference on Small Satellites.

[14] Jobanputra, Rishi (2000) "Report on Helmholtz Coils"; Canadian Space Agency, Space Technologies Division, QuickSat Development Project.

[15] Kalman, R.E.(1960) "A new Approach to Linear Filtering and Prediction Problems"; ASME Journal of Basic Engineering 82(Series D)pp.35-45.

[16] Laderman, M. (2002) "RACE Ryerson Satellite Attitude Control Experiment"; Ryerson University

[17] Lefferts, E.J., Markley, F.L, Shuster, M.D. (1982) "Kalman Filtering for Spacecraft Attitude Estimation", Guidance Volume 5, No.5.

[18] Marchetti, Stephen (1999) "Design and Development of the Tri-Axial Helmholtz Coil Assembly for the Quicksat HITL"; Canadian Space Agency, Space Technologies Division, Quicksat Development Project, October.

[19] McTavish (2003) "Ryerson Attitude Control Experiment, Software Design Specification/Description, Draft"; Internal Report.

[20] McTavish (2003) "Ryerson Attitude Control Experiment, System Modeling, Draft"; Internal Report.

[21] Ng, Alfred (2003) "Future Generation of Nanosatellites"; Presentation for Canadian Space Agency Technology Days.

[22] Nise, Norman (2000) Control Systems Engineering, Third Edition; John Wiley \& Sons, Inc, New York, US.

[23] Pastena, M and Grassi, M (2000) "Robust Control of the Smart Microsatellite Attitude Dynamics"; Acta Astronautica Vol 47, Nos 2-9, pp. 119-128. 
[24] von Shéele, F (2003) "The Odin Project — Lessons for a Follow-On Earth Observation Mission"; Acta Astronautic Volume 53 pp. 739-747.

[25] Sidi, Marcel (1997)Spacecraft Dynamics and Control A Practical Engineering Approach; Cambridge University Press.

[26] (1999) Thomsen et al "Danish Orsted Mission In-Orbit Experiences and Status of The Danish Small Satellite Programme"; Paper number SSC99-I-8, 13th Annual AIAA/USU Conference on Small Satellites.

[27] Wertz, J.R. (1976)Spacecraft Attitude Dynamics and Control; Kluwer, pp. 820.

[28] Wisniewski, Rafal and Blanke, Mogens (1999) "Fully magnetic attitude control for spacecaft subject to gravity gradient";Automatica 35, pp. 1201-1214. 